\title{
Distributed Synchronization in Networks of Agent Systems With Nonlinearities and Random Switchings
}

\author{
Yang Tang, Member, IEEE, Huijun Gao, Senior Member, IEEE, Wei Zou, and Jürgen Kurths
}

\begin{abstract}
In this paper, the distributed synchronization problem of networks of agent systems with controllers and nonlinearities subject to Bernoulli switchings is investigated. Controllers and adaptive updating laws injected in each vertex of networks depend on the state information of its neighborhood. Three sets of Bernoulli stochastic variables are introduced to describe the occurrence probabilities of distributed adaptive controllers, updating laws and nonlinearities, respectively. By the Lyapunov functions method, we show that the distributed synchronization of networks composed of agent systems with multiple randomly occurring nonlinearities, multiple randomly occurring controllers, and multiple randomly occurring updating laws can be achieved in mean square under certain criteria. The conditions derived in this paper can be solved by semi-definite programming. Moreover, by mathematical analysis, we find that the coupling strength, the probabilities of the Bernoulli stochastic variables, and the form of nonlinearities have great impacts on the convergence speed and the terminal control strength. The synchronization criteria and the observed phenomena are demonstrated by several numerical simulation examples. In addition, the advantage of distributed adaptive controllers over conventional adaptive controllers is illustrated.
\end{abstract}

Index Terms-Bernoulli stochastic variables, complex dynamical networks, distributed synchronization, multi-agent systems, multiple random nonlinearities, multiple random updating laws.

Manuscript received February 27, 2012; revised June 6, 2012; accepted June 16, 2012. Date of publication August 6, 2012; date of current version January 11, 2013. This research is supported by 973 Project (2009CB320600), the National Natural Science Foundation of China (60825303, 60834003, 61021002, 11147179) and the Key Laboratory of Integrated Automation for the Process Industry (Northeastern University), the Fundamental Research Funds for the Central Universities of China (2011QN161), SUMO (EU), IRTG 1740 (DFG) and the Alexander von Humboldt Foundation of Germany. This paper was recommended by Associate Editor G. Dimirovski.

Y. Tang is with the Research Institute of Intelligent Control and Systems, Harbin Institute of Technology, Harbin 150080, China and Institute of Physics, Humboldt University Berlin, Berlin 12489, Germany and Potsdam Institute for Climate Impact Research, Potsdam 14415, Germany (e-mail: tangtany @ gmail.com).

H. Gao is with the Research Institute of Intelligent Control and Systems, Harbin Institute of Technology, Harbin 150080, China (e-mail: hjgao@ hit.edu.cn).

W. Zou is with the School of Mathematics and Statistics, Huazhong University of Science and Technology, Wuhan 430074, China, Institute of Physics, Humboldt University Berlin, Berlin 12489, Germany and Potsdam Institute for Climate Impact Research, Potsdam 14415, Germany (e-mail: zouwei2010@mail.hust.edu.cn).

J. Kurths is with the Potsdam Institute for Climate Impact Research, Potsdam, Germany and Institute of Physics, Humboldt University, 10099 Berlin, Germany and Institute for Complex Systems and Mathematical Biology, University of Aberdeen, AB24 3UE Aberdeen, U.K. (e-mail: Juergen.Kurths @ pik-potsdam.de).

Color versions of one or more of the figures in this paper are available online at http://ieeexplore.iee.org.

Digital Object Identifier 10.1109/TSMCB.2012.2207718

\section{INTRODUCTION}

$\mathbf{R}$ ECENTLY, cooperative collective behavior in networks of autonomous agents has attracted growing attention owing to an increasing interest in understanding intriguing animal group behaviors, such as flocking and swarming, and also due to their emerging broad applications to sensor networks and unmanned air vehicles formations [1]. To coordinate with the agents in a networked environment, agents are required to share information with their adjacent neighbors and thus to achieve an agreement. As one of the mostly investigated dynamical behaviors in the field of information consensus and complex networks, synchronization/consensus of multi-agent systems or complex networks has drawn significant research interest, and a great number of results have been reported; e.g. [2]-[15] and references therein.

Usually, synchronization in networks of coupled systems can also be referred as the consensus problem of multi-agent networked systems from the perspective of control theory and engineering application [16]-[27]. Distributed algorithms have attracted increasing attention in filtering of sensor networks [14], and gossip algorithms [28]. In [20], a unified framework is introduced to address the consensus of multi-agent systems and the synchronization of complex networks. In [29], a second-order consensus problem for multi-agent systems with nonlinear dynamics and directed topologies is investigated. In [30], the relationship between Lipschitz-like assumptions is compared, and the application of these assumptions is used to synchronization or consensus of complex networks.

In networked systems, networks of multi-agent systems are often subject to a random environment [31], [32] and hence, the stochastic modeling issue has been of vital significance in many branches of science such as neurotransmitters and packet dropouts. The corresponding random phenomena can be considered in random switching ways, which have been paid considerable research attention in the literature. For instance, in [33], sufficient conditions for asymptotic almost sure consensus are presented for the case of positive weights and for the case of arbitrary weights, where the existence of any edge is probabilistic and independent of the existence of any other edge. In [34], the global synchronization problem has been investigated for a complex network with blinking connections which are randomly switched on and off with a given probability, and the frequency of switching is high compared to the network dynamics. In particular, in [32], the concept of randomly occurred nonlinearities (RONs) is proposed to explain nonlinear disturbances in networked control systems and biological networks. The filtering problem of randomly occurred phenomena in networked systems was investigated [35], [36]. The problem of stochastic synchronization analysis for complex networks 
composed of coupled discrete-time neural networks with RONs and time delays was studied. Unfortunately, the RONs considered in [32] are confined to two cases, and multiple RONs (MRONs) [35] have not been introduced in investigating the synchronization of networks of agent systems and the impact of MRONs on synchronization performance is still open, which is the first motivation of this paper.

In addition, most of the aforementioned results concerning synchronization of complex networks or consensus of multiagent systems are based on the implicit assumption that the communications between the node dynamics and controller are perfect. In many practical applications, however, there may be a nonzero probability that some measurements or control inputs will be lost during their transmission. Usually, there exist two approaches to describe the imperfect communications among networks or multi-agent systems [31]. The first one is to use the deterministic switching method to characterize the control failure in networked systems [37]-[40]. Among them, in [40], an effective approach was proposed to analyze the delayed neural networks under arbitrary switching with average dwell time and presented a less conservative sufficient stability condition. The approaches in [40] can also be utilized to study the synchronization problem for a linearly coupled array of networked systems, and can improve some existing results. The second one is to utilize stochastic modeling analysis to describe the intermittent communications in the networked systems [31], [41], [42]. Note that utilizing Bernoulli variables is the most common way to describe packet losses [31], [43], [44], random sensor delays [45], and unreliable control inputs [46] due to their simplicities and efficiency. Unfortunately, another important random phenomenon, i.e., randomly occurring updating laws (ROULs) in adaptive controllers, is widely neglected in the existing literature. ROULs are similar to randomly occurring control, which take place in environments suffering from packet dropouts and data missing, and thus the feedback information of updating laws are lost in signals transmission of networked systems. To the best of our knowledge, there has not been any work being reported in the literature concerning synchronization or consensus problems in the context of networked systems where the controllers or updating laws are described by sets of Bernoulli variables, which is the second motivation of this paper.

Control cost and convergence speed of networked dynamics are two important measures to define the performance of networked systems [19], [28], [31]. In [47], the problem of finding the edge weights that result in the least mean-square deviation in a steady state of a stochastic model for distributed average consensus is investigated. In [48], synchronization of complex networks is studied, and the bounds of convergence speed and control cost are provided. However, up to now, the impacts of sets of Bernoulli variables on synchronization or consensus of networked systems have not been addressed yet, and the purpose of this paper is therefore to shorten such a gap, which is the third motivation of this paper.

In this paper, the distributed synchronization problem is studied for networks of agent systems with MRONs, multiple randomly occurring controllers (MROCs), and multiple randomly occurring updating laws (MROULs). The controllers and the adaptive updating laws injected in each vertex of networks are dependent on the state information of its nearest neighborhood. By Lyapunov functions method, the distributed synchronization of networks of agent systems with MRONs, MROCs, and MROULs can be achieved in mean square. The main merits of this paper are the following ones: 1) the concept of MRONs is used and is taken into account to model networks of agent systems, hence reflecting the reality more closely; 2) MROCs and MROULs are considered to describe unreliable stochastic effects in multi-agent systems simultaneously; 3 ) the effects of occurrence probabilities of MRONs, MROCs, and MROULs on control cost and convergence speed are illustrated; 4) the algorithm developed is new in terms of semidefinite programming (SDP), which can be efficiently solved by Yamlip [49].

The organization of this paper is illustrated as follows. Section II proposes networks model composed of agent systems with MRONs, MROCs, and MROULs, and outlines some preliminaries. In Section III, by using stochastic analysis techniques and graph theory, we present sufficient conditions in terms of SDP to ensure the synchronization of the addressed networks of agent systems in the mean square. The upper bounds of synchronization errors and control gains are also presented. In Section IV, several numerical examples are provided to show the applicability of the obtained results. The conclusions are finally drawn in Section V.

\section{PReliminaries}

Notations: In this paper, $\mathbb{R}^{n}$ and $\mathbb{R}^{n \times m}$ represent, respectively, the $n$-dimensional Euclidean space and the set of all real matrices. The superscript " $T$ " stands for matrix transposition, and the notation $X \geq Z$ (respectively, $X>Z$ ) where $X$ and $Z$ are symmetric matrices indicates that $X-Z$ is positive semi-definite (respectively, positive definite). $I_{n}$ is the identity matrix with order $n . \mathbb{E}\{x\}$, and $\mathbb{E}\{x \mid z\}$ denote the expectation of $x$ and the expectation of $x$ conditional on $z$. $\|\cdot\|$ denotes the Euclidean vector norm in $\mathbb{R}^{n}$. The Kronecker product of matrices $X \in \mathbb{R}^{m \times n}$ and $Z \in \mathbb{R}^{p \times q}$ is a matrix in $\mathbb{R}^{m p \times n q}$ and represented as $X \otimes Z \cdot \lambda_{\min }(\cdot)$ and $\lambda_{\max }(\cdot)$ stand for the minimum and maximum eigenvalue of a matrix. Let a graph be $\mathcal{G}=[\mathcal{V}, \mathcal{E}]$, where $\mathcal{V}=\{1, \ldots, N\}$ is the vertex set and $\mathcal{E}=\{e(i, j)\}$ the edge set. $\mathcal{N}(i)$ means the neighborhood of vertex $i$ in the sense $\mathcal{N}(i)=\{j \in\{\mathcal{V}: e(i, j) \in \mathcal{E}\}$. Here, graph $\mathcal{G}$ is supposed to be connected, unweighted and undirected, $(e(i, j) \in \mathcal{E}$ implies $e(j, i)) \in \mathcal{E}$ and simple (without self-loops and multiple edges). Let $L=\left[a_{i j}\right]_{i, j=1}^{N}$ be the Laplacian matrix of graph $\mathcal{G}$, which is defined as: for any pair $i \neq j, a_{i j}=a_{j i}=-1$ if $e(i, j) \in \mathcal{E}$; otherwise, $a_{i j}=a_{j i}=0$. $a_{i i}=-\sum_{j=1, j \neq i}^{N} a_{i j}$ represents the degree of vertex $i(i \in \mathcal{V})$.

Here, the network of agent systems is considered

$$
d x_{i}(t)=\left[f\left(x_{i}, t\right)+D \sum_{j \in \mathcal{N}(i)}\left(x_{j}(t)-x_{i}(t)\right)\right] d t, \quad i \in \mathcal{V}
$$

where $\quad x_{i}(t)=\left[x_{i 1}(t), x_{i 2}(t), \ldots, x_{i n}(t)\right]^{T} \in \mathbb{R}^{n}(i \in \mathcal{V})$ is the state vector of the $i$ th vertex and $f\left(x_{i}, t\right)=$ $\left[f_{1}\left(x_{i}, t\right), \ldots, f_{n}\left(x_{i}, t\right)\right]^{T}$ is a continuous vector function, which occurs according to the occurrence probabilities. $D$ represents the coupling strength of the network. From the Gershgorin disk theorem, all the eigenvalues of $L$ 
corresponding to graph $\mathcal{G}$ satisfy the following relationship $0=\lambda_{1}(L) \leq \lambda_{2}(L) \leq \ldots \leq \lambda_{N}(L)$. In addition, $\mathcal{G}$ is connected if and only if $\lambda_{2}(L)>0$; namely, $L$ is irreducible. The nonlinearity $f($.$) takes values in a finite set, i.e.,$ $f(.) \in\left\{g_{1}(),. \ldots, g_{q}().\right\}$. $\mathcal{I}$ denotes the set $\mathcal{I}=\{1, \ldots, q\}$. The nonlinearity $g_{m}($.$) is assumed to be i. i. d. (independent$ and identically distributed), with the probabilities given by

$$
\operatorname{Prob}\left\{f(.)=g_{m}(.)\right\}=\kappa_{m}, \quad m \in \mathcal{I}
$$

where $\kappa_{m}$ is a positive scalar and $\sum_{m \in \mathcal{I}} \kappa_{m}=1$. The network (1) is a basic model to study synchronization of networks of agent systems for rather general cases particularly when the synchronized state is a time-varying function and switching rather than a constant equilibrium [50].

By introducing stochastic variables $\kappa_{m}(t)$ to represent the occurrence of nonlinearities, where $\mathbb{E}\left\{\kappa_{m}(t)\right\}=\kappa_{m}$, we can also rewrite the system (1) as follows:

$$
d x_{i}(t)=\left[\sum_{m \in \mathcal{I}} \kappa_{m}(t) g_{m}\left(x_{i}, t\right)-D \sum_{j=1}^{N} a_{i j} x_{j}(t)\right] d t, i \in \mathcal{V} .
$$

Remark 1: RONs are considered to model a complex network in [32], and its synchronization was studied. According to [45] and [35], in this paper, a finite set is introduced to describe the multiple random occurrence of nonlinearities. Utilizing such an expression is more general than RONs and is able to describe multiple nonlinearities cases in a stochastic way. Here, we can regard the dynamics of each node/vertex/agent in networks switches from a finite set according to the probabilities of $\kappa_{m}$.

Our aim is to develop distributed controllers to achieve synchronization of the network of agent systems (1) or (3) in mean square. Therefore, distributed controllers $u_{i}(t)$ are injected to the vertex set

$$
\begin{aligned}
d x_{i}(t)=\left[\sum_{m \in \mathcal{I}} \kappa_{m}(t) g_{m}\left(x_{i}, t\right)+D \sum_{j \in \mathcal{N}(i)}\right. \\
\\
\left.\quad \times\left(x_{j}(t)-x_{i}(t)\right)+\eta_{i}(t) u_{i}(t)\right] d t, \quad i \in \mathcal{V}
\end{aligned}
$$

where $\eta_{i}(t), i \in \mathcal{V}$, is a Bernoulli stochastic variable that describes the following random events for the network of agent systems (4):

$$
\begin{cases}\text { Event } 1: & \text { vertex } i \text { in (4) experiences } u_{i}(t) \\ \text { Event 2: } & \text { vertex } i \text { in (4) does not experience } u_{i}(t)\end{cases}
$$

which shows that the controllers might fail to work due to the existence of packet dropouts.

The distributed controller is represented as follows:

$$
u_{i}(t)=\sum_{j \in \mathcal{N}(i)} \epsilon_{i}(t)\left(x_{j}(t)-x_{i}(t)\right), \quad i \in \mathcal{V}
$$

where $\epsilon_{i}(t)$ is the control gain of vertex $i$, which is updated according to updated laws. $\eta_{i}(t)$ is defined by

$$
\eta_{i}(t)= \begin{cases}1, & \text { if Event } 1 \text { occurs } \\ 0, & \text { if Event } 2 \text { occurs }\end{cases}
$$

where $\mathbb{E}\left\{\eta_{i}(t)\right\}=\eta_{i} \in[\check{\eta}, \hat{\eta}] \subseteq[0,1]$. The main feature of distributed controllers/algorithms/protocals is that a vertex can utilize the information of its neighbors with high efficiency but does not need the information of entire networks [19].

Remark 2: The practical controllers are influenced by environmental circumstances [31], [37]. The controller failure or inactivation arises from two aspects. The first reason is that the signals are not transmitted perfectly or the controller itself is not available, e.g., as in the case of the packet dropout phenomenon, intermittent communication, and repairs of the actuator [31], [46]. The other is a positive reason: for an economic or system life consideration, where the controller is suspended from time to time [37]. Here, a set of Bernoulli variables are used to describe the controller failure or controller suspension.

The control gain $\epsilon_{i}(t)$ in (6) is adjusted according to the following MROULs:

$$
\begin{aligned}
d \epsilon_{i}(t)=\xi_{i}(t) \alpha_{i} & {\left[\sum_{j \in \mathcal{N}(i)}\left(x_{j}(t)-x_{i}(t)\right)\right]^{T} } \\
& \times\left[\sum_{j \in \mathcal{N}(i)}\left(x_{j}(t)-x_{i}(t)\right)\right] d t, \quad i \in \mathcal{V}
\end{aligned}
$$

where $\alpha_{i} \geq 0$ and $\xi_{i}(t)$ is a stochastic variable that is independent of $\eta_{i}(t)$ and indicates the following random events:

$$
\begin{cases}\text { Event } 3: & \text { (6) experiences (8) } \\ \text { Event } 4: & \text { (6) does not experience (8). }\end{cases}
$$

Here, $\xi_{i}(t)$ is defined by

$$
\xi_{i}(t)= \begin{cases}1, & \text { if Event } 3 \text { occurs, } \\ 0, & \text { if Event } 4 \text { occurs }\end{cases}
$$

where $\mathbb{E}\left\{\xi_{i}(t)\right\}=\xi_{i} \in[\check{\xi}, \hat{\xi}] \subseteq[0,1]$. In this paper, three sets of Bernoulli variables, i.e., $\kappa_{m}(t), \eta_{i}(t)$ and $\xi_{i}(t)$, are introduced. We assume that all the Bernoulli variables are independent of each other. Also, the Bernoulli variables here follow an unknown, but exponential distribution of switchings and the probabilities should be known a priori [51].

Two measures are employed to characterize the performance of synchronization. One is the average of terminal control strengths, which is formulated as follows:

$$
\epsilon=\frac{1}{N} \sum_{i \in \mathcal{V}} \epsilon_{i, \infty}(i \in \mathcal{V})
$$

where $\epsilon_{i, \infty}=\lim _{t \rightarrow \infty} \epsilon_{i}(t)$. The other measure is the convergence rate, which is defined as follows:

$$
\gamma=\int_{0}^{\infty} \frac{1}{N-1} \sum_{i \in \mathcal{V}}\left[x_{i}(t)-\bar{x}(t)\right]^{T}\left[x_{i}(t)-\bar{x}(t)\right] d t
$$

where $\bar{x}(t)=(1 / N) x(t)$. As shown in (11) and (12), it can be found that a good synchronization performance means a high convergence rate (a small $\gamma$ ) and low control cost (a small $\epsilon$ ). 
Remark 3: Note that if $\eta_{i}=\eta$ or $\xi_{i}=\xi$, the occurrence probabilities of controllers and updating laws are identical in the nodes of the whole networks. Such a simple assumption, however, does have its limitations since it cannot cover some practical cases where multiple control failures or suspensions occur. Like the idea of multiple missing measurements [52], in this paper, we consider MROCs and MROULs. Note that the implementation of controllers are not subject to stochastic effects. However, the transmission of control signals in networked circumstances suffers from packet dropouts. For the sake of simplicity, we name this kind of controllers as MROCs. Therefore, the assumption is relaxed, and in each vertex of the network, the unreliable implementation probabilities of the controllers and updating laws are different.

Remark 4: If $\eta_{i}$ and $\xi_{i}$ are both equal to 1 , controllers and updating rules will be reduced to the usual studied controllers and updating laws, which indicates that controllers and updating laws are always available. If $\eta_{i}=1$ and $\xi_{i}=0$, controllers are available, and control strengths do not update. If $\eta_{i}=0$ and $\xi_{i}=1$, controllers are not available and control strengths can update. If $\eta_{i}=0$ and $\xi_{i}=0$, the problem is reduced to the synchronization problem without controllers. The problem of such models was widely studied in the literature, see [4] and references therein. Also, the control and updating failure in the considered system can also be modeled by Markovian switching [8] and switching signals [39], which would be more general than our model.

Remark 5: Compared with [32], [48], [52], the problem considered here is to study the distributed synchronization of networks of agent systems with multiple random failures existing in controllers and updating laws. The model is general to include several switchings in dynamics of each agent and packet dropouts, and therefore our model can describe realworlds networks well. In addition, criteria are shown in terms of SDP, and the effects of stochastic variables on synchronization performance are illustrated in both theoretical and simulation ways.

Before presenting our main results, the following assumptions and definitions are required.

Assumption 1: [53] Suppose that vector-valued continuous functions $g_{m}(x, t): \mathbb{R}^{n} \times \mathbb{R}^{+} \rightarrow \mathbb{R}^{n}$ are said to be uniformly decreasing if there exist $\theta_{m}>0 \in \mathbb{R}$ and $\Delta_{m}>0 \in \mathbb{R}$ such that

$$
\begin{aligned}
(x-y)^{T}\left[g_{m}(x, t)\right. & \left.-g_{m}(y, t)-\theta_{m}(x-y)\right] \\
& \leq-\Delta_{m}(x-y)^{T}(x-y), \quad m \in \mathcal{I}
\end{aligned}
$$

holds for all $x, y \in \mathbb{R}^{n}$ and $t \geq 0$.

Note that the relationship between Assumption 1 and Lipchitz condition is revealed in [30].

Assumption 2: [32] $g_{m}\left(x_{i}, t\right)$ is said to be locally uniformly Lipschitz continuous with respect to $t$ if there exist positive constants $\varphi_{m}$ such that the following inequalities hold for all $x_{i}, x_{j} \in \mathbb{R}^{n}$ :

$$
\left\|g_{m}\left(x_{i}, t\right)-g_{m}\left(x_{j}, t\right)\right\| \leq \varphi_{m}\left\|x_{i}-x_{j}\right\|, i, j \in \mathcal{V}, m \in \mathcal{I} .
$$

Assumption 3: $g_{m}(0, t)=0$.

Definition 1: Let $x_{i}\left(t ; t_{0}, X_{0}\right)(1 \leq i \leq N)$ be a solution of the array of the stochastic complex network (1), where $X_{0}=$ $\left(x_{1}^{0}, x_{2}^{0}, \ldots, x_{N}^{0}\right)$. If there is a nonempty subset $\Omega \subseteq \mathbb{R}^{n}$, with $x_{i}^{0} \in \Omega(1 \leq i \leq N)$, such that $x_{i}\left(t ; t_{0}, X_{0}\right) \in \mathbb{R}^{n}$ for all $t \geq$ $t_{0}, 1 \leq i \leq N$, and

$$
\lim _{t \rightarrow \infty} \mathbb{E} \sum_{i \in \mathcal{V}}\left\|x_{i}(t)-x_{j}(t)\right\|^{2}=0, \quad i, j \in \mathcal{V}
$$

then the network of agent systems (1) is said to achieve synchronization in mean square under the distributed controller (6).

\section{Main Results}

In this section, the synchronization problem of the network of agent systems with MRONs (1) is studied in the sense of mean square via MROCs and MROULs. The synchronization criteria are given as follows.

\section{A. Criteria for Synchronization of Networks of Agent Systems With Multiple Random Controllers and Nonlinearities}

Theorem 1: Suppose that $g_{m}(x, t),(m \in \mathcal{I})$ is continuous on $(x, t) \in \mathbb{R}^{n} \times \mathbb{R}^{+}$, satisfies Assumptions 1 , 2, and 3, graph $\mathcal{G}$ is connected, $\mathcal{H}=\operatorname{diag}\left\{\eta_{1}, \ldots, \eta_{N}\right\}$ and $c$ is a positive constant, which makes the following inequality hold:

$$
\left(\sum_{m \in \mathcal{I}} \kappa_{m} \theta_{m} I_{N}-c L \mathcal{H}-D L\right) L \leq 0
$$

for any initial data $x_{i}(0) \in \mathbb{R}^{n}, i \in \mathcal{V}$, then system (4) under (6) and (8) will be globally synchronized in mean square, and the coupling strengths will converge, i.e., $\lim _{t \rightarrow \infty} \epsilon_{i}(t)=\epsilon_{i, \infty}$, where $\epsilon_{i, \infty}(i \in \mathcal{V}) \in \mathbb{R}$ is a constant.

Proof: Define $x=\left[x_{1}^{T}, \ldots, x_{N}^{T}\right]^{T} \in \mathbb{R}^{n N}$. Let $e_{i j}=$ $x_{i}-x_{j}, \forall i, j \in \mathcal{V}$. Note that $\lambda_{2}(L)>0$ since graph $\mathcal{G}$ is connected and $L$ is irreducible. Since $L$ is irreducible, we conclude that $\left(L \otimes I_{n}\right) x=0$ if and only if $e_{i j}=0$ holds for $\forall i, j \in \mathcal{V}$.

The network of agent systems (3) with Bernoulli variables is a special case of stochastic systems with Markovian switching [51]. Thus, the existence and uniqueness of solutions of (3) can be solved by the theory of stochastic systems with Markovian switching [54].

Consider the following Lyapunov candidate:

$$
V(t)=\frac{1}{4} \sum_{i \in \mathcal{V}} \sum_{j \in \mathcal{N}(i)} e_{i j}^{T} e_{i j}+\sum_{i \in \mathcal{V}} \frac{\eta_{i}}{2 \alpha_{i} \xi_{i}}\left(\epsilon_{i}(t)-c\right)^{2}
$$

where $c$ is a positive constant.

The operator $\mathcal{L}$ is defined as follows [55]:

$$
\mathcal{L} V(t)=\lim _{\phi \rightarrow 0^{+}} \frac{1}{\phi}\{\mathbb{E}(V(t+\phi) \mid t)-V(t)\} .
$$

Then, we can obtain from (4) and (6)

$$
\begin{aligned}
\mathcal{L} V(t)= & \frac{1}{2} \sum_{i \in \mathcal{V}} \sum_{j \in \mathcal{N}(i)} e_{i j}^{T} \\
& \times\left\{\begin{array}{l}
\sum_{m \in \mathcal{I}} \kappa_{m}(t) \times\left(g_{m}\left(x_{i}, t\right)-g_{m}\left(x_{j}, t\right)\right) \\
\\
\quad+D \sum_{k \in \mathcal{N}(i)}\left(x_{k}-x_{i}\right)-D \sum_{l \in \mathcal{N}(j)}\left(x_{l}-x_{j}\right)
\end{array}\right.
\end{aligned}
$$




$$
\begin{aligned}
& +\eta_{i}(t) \epsilon_{i}(t)\left[\sum_{k \in \mathcal{N}(i)}\left(x_{k}-x_{i}\right)\right] \\
& \left.-\eta_{j}(t) \epsilon_{j}(t)\left[\sum_{l \in \mathcal{N}(j)}\left(x_{l}-x_{j}\right)\right]\right\} \\
& +\sum_{i \in \mathcal{V}} \frac{\eta_{i}}{\xi_{i}} \xi_{i}(t)\left(\epsilon_{i}(t)-c\right)\left[\sum_{j \in \mathcal{N}(i)} e_{i j}\right]^{T}\left[\sum_{j \in \mathcal{N}(i)} e_{i j}\right] .
\end{aligned}
$$

From the definitions of $e_{i j}, x, L$ and taking expectations of $\eta_{i}(t)$ and $\xi_{i}(t)$, the following equalities hold:

$$
\begin{aligned}
& \frac{1}{2} \sum_{i \in \mathcal{V}} \sum_{j \in \mathcal{N}(i)} e_{i j}^{T} e_{i j}=x^{T}\left(L \otimes I_{n}\right) x \\
& \sum_{i \in \mathcal{V}}\left(\sum_{j \in \mathcal{N}(i)} e_{i j}\right)^{T}\left(\sum_{j \in \mathcal{N}(i)} e_{i j}\right) \\
& \quad=x^{T}\left(L^{2} \otimes I_{n}\right) x \\
& \mathbb{E}\left\{\sum_{i \in \mathcal{V}} \sum_{j \in \mathcal{N}(i)} e_{i j}^{T} \eta_{i}(t) \epsilon_{i}(t) \sum_{k \in \mathcal{N}(i)} e_{k i}\right\} \\
& \quad=-\sum_{i \in \mathcal{V}} \eta_{i} \epsilon_{i}(t)\left[\sum_{j \in \mathcal{N}(i)} e_{i j}\right]^{T}\left[\sum_{j \in \mathcal{N}(i)} e_{i j}\right] \\
& \mathbb{E}\left\{\sum_{i \in \mathcal{V}} \frac{\eta_{i}}{\xi_{i}} \xi_{i}(t)\left(\epsilon_{i}(t)-c\right)\left[\sum_{j \in \mathcal{N}(i)} e_{i j}\right]^{T}\left[\sum_{j \in \mathcal{N}(i)} e_{i j}\right]\right\} \\
& \quad=\mathbb{E}\left\{\sum_{i \in \mathcal{V}} \eta_{i}\left(\epsilon_{i}(t)-c\right)\left[\sum_{j \in \mathcal{N}(i)} e_{i j}\right]^{T}\left[\sum_{j \in \mathcal{N}(i)} e_{i j}\right]\right\} .
\end{aligned}
$$

Then, using the inequality (16) in Theorem 1, Assumptions 1 and 2, (19) and (20), we have

$$
\begin{aligned}
\mathbb{E} \mathcal{L} V(t)= & \mathbb{E} \frac{1}{2} \sum_{i \in \mathcal{V}} \sum_{j \in \mathcal{N}(i)} e_{i j}^{T} \\
& \times\left\{\sum_{m \in \mathcal{I}} \kappa_{m}(t)\left[g_{m}\left(x_{i}, t\right)-g_{m}\left(x_{j}, t\right)-\theta_{m} e_{i j}\right]\right\} \\
& +\frac{1}{2} \sum_{i \in \mathcal{V}} \sum_{j \in \mathcal{N}(i)} \sum_{m \in \mathcal{I}} \kappa_{m}(t) \theta_{m} e_{i j}^{T} e_{i j} \\
& -D \sum_{i \in \mathcal{V}}\left[\sum_{j \in \mathcal{N}(i)} e_{i j}\right]^{T}\left[\sum_{j \in \mathcal{N}(i)} e_{i j}\right] \\
& -c \sum_{i \in \mathcal{V}} \eta_{i}\left[\sum_{j \in \mathcal{N}(i)} e_{i j}\right]^{T}\left[\sum_{j \in \mathcal{N}(i)} e_{i j}\right] \\
\leq & \mathbb{E}-\frac{1}{2} \sum_{I \in \mathcal{V}} \sum_{j \in \mathcal{N}(i)} \sum_{m \in \mathcal{I}} \kappa_{m} \Delta_{m} e_{i j}^{T} e_{i j}
\end{aligned}
$$

$$
\begin{aligned}
& +\frac{1}{2} \sum_{I \in \mathcal{V}} \sum_{j \in \mathcal{N}(i)} \sum_{m \in \mathcal{I}} \kappa_{m} \theta_{m} e_{i j}^{T} e_{i j} \\
& -D \sum_{i \in \mathcal{V}}\left[\sum_{j \in \mathcal{N}(i)} e_{i j}\right]^{T}\left[\sum_{j \in \mathcal{N}(i)} e_{i j}\right] \\
& -c \sum_{i \in \mathcal{V}} \eta_{i}\left[\sum_{j \in \mathcal{N}(i)} e_{i j}\right]^{T}\left[\sum_{j \in \mathcal{N}(i)} e_{i j}\right] \\
& =\mathbb{E} x^{T}\left[\left(\sum_{m \in \mathcal{I}} \kappa_{m}\left(\theta_{m}-\Delta_{m}\right) I_{N}-c L \mathcal{H}-D L\right) L \otimes I_{n}\right] x \\
& \leq \mathbb{E} x^{T}\left(-\sum_{m \in \mathcal{I}} \kappa_{m} \Delta_{m} L \otimes I_{n}\right) x
\end{aligned}
$$

where $\mathcal{H}=\operatorname{diag}\left\{\eta_{1}, \ldots, \eta_{N}\right\}$.

Hence, synchronization will be achieved in mean square. This completes the proof.

Remark 6: As seen from Theorem 1, the occurrence probabilities of various nonlinearities $\kappa_{m}$, the forms of nonlinearities $\theta_{m}$, and the occurrence probabilities of controllers $\eta_{i}$ have strong effects on ensuring the synchronization performance of networks of agent systems. Therefore, the conditions developed in this paper are probability-dependent or nonlinearitydependent criteria.

Corollary 1: Assume that only one nonlinearity exists in the network of agent systems (1), i.e., $m \in \mathcal{I}=\{1\}$. Suppose that $g_{1}(x, t)$ is continuous on $(x, t) \in \mathbb{R}^{n} \times \mathbb{R}^{+}$, satisfies Assumptions 1,2 , and 3 , graph $\mathcal{G}$ is connected and $c$ is a positive constant, which makes the following inequality hold:

$$
\left(\theta_{1} I_{N}-c L \mathcal{H}-D L\right) L \leq 0
$$

for any initial data $x_{i}(0) \in \mathbb{R}^{n}, i \in \mathcal{V}$, then the network of agent systems (4) under (6) and (8) will be globally synchronized in mean square, and the coupling strengths will converge, i.e., $\lim _{t \rightarrow \infty} \epsilon_{i}(t)=\epsilon_{i, \infty}$, where $\epsilon_{i, \infty}(i \in \mathcal{V}) \in \mathbb{R}$ is a constant.

If the occurrence probabilities of MROCs are equal in all the vertices, we have the following results.

Corollary 2: Suppose that $g_{m}(x, t),(m \in \mathcal{I})$ is continuous on $(x, t) \in \mathbb{R}^{n} \times \mathbb{R}^{+}$, satisfies Assumptions 1,2 , and 3, graph $\mathcal{G}$ is connected, $\eta_{i}=\eta(i \in \mathcal{V})$ and $c$ is a positive constant, which makes the following inequality hold:

$$
\left(\sum_{m \in \mathcal{I}} \kappa_{m} \theta_{m} I_{N}-c \eta L-D L\right) L \leq 0
$$

then the network of agent systems (4) under (6) and (8) will be globally synchronized in mean square, and the coupling strengths will converge for any initial data $x_{i}(0) \in \mathbb{R}^{n}, i \in \mathcal{V}$, i.e., $\lim _{t \rightarrow \infty} \epsilon_{i}(t)=\epsilon_{i, \infty}$, where $\epsilon_{i, \infty}(i \in \mathcal{V}) \in \mathbb{R}$ is a constant.

Furthermore, the following corollary can be obtained.

Corollary 3: Suppose that $g_{m}(x, t),(m \in \mathcal{I})$ is continuous on $(x, t) \in \mathbb{R}^{n} \times \mathbb{R}^{+}$, satisfies Assumptions 1, 2, and 3, graph $\mathcal{G}$ is connected, $\eta_{i}=\eta(i \in \mathcal{V})$ and $c$ is a positive constant, which makes the following inequality hold:

$$
\sum_{i=2}^{N}\left[\sum_{m \in \mathcal{I}} \kappa_{m}\left(\theta_{m}-\Delta_{m}\right)-c \eta \lambda_{i}(L)-D \lambda_{i}(L)\right]<0
$$


then the network of agent systems (4) under (6) and (8) will be globally synchronized in mean square, and the coupling strengths will converge for any initial data $x_{i}(0) \in \mathbb{R}^{n}, i \in \mathcal{V}$, i e., $\lim _{t \rightarrow \infty} \epsilon_{i}(t)=\epsilon_{i, \infty}$, where $\epsilon_{i, \infty}(i \in \mathcal{V}) \in \mathbb{R}$ is a constant.

Proof: According to (21) of Theorem 1

$\mathbb{E} \mathcal{L} V(t) \leq \mathbb{E} x^{T}\left[\left(\sum_{m \in \mathcal{I}} \kappa_{m}\left(\theta_{m}-\Delta_{m}\right) I_{N}-c \eta L-D L\right) \times L \otimes I_{n}\right] x$.

From the matrix decomposition theory [56], there exists a unitary matrix $U$ such that $L=U \Lambda U^{T}$, where $\Lambda=\operatorname{diag}$ $\left\{\lambda_{1}(L), \lambda_{2}(L), \ldots, \lambda_{N}(L)\right\}=\operatorname{diag}\left\{0, \lambda_{2}(L), \ldots, \lambda_{N}(L)\right\}$, $U=\left[u_{1}, u_{2}, \ldots, u_{N}\right]$, and $u_{1}=1 / \sqrt{N}[1,1, \ldots, 1]^{T}$. Let $z(t)=\left(U^{T} \otimes I_{n}\right) x(t)=\left[z_{1}^{T}(t), z_{2}^{T}(t), \ldots, z_{N}^{T}(t)\right]^{T}, \quad$ where $z_{i}(t) \in \mathbb{R}^{n}(i \in \mathcal{V})$. Therefore, it follows that:

$$
\begin{aligned}
x^{T} & {\left[\sum_{m \in \mathcal{I}} \kappa_{m}\left(\theta_{m}-\Delta_{m}\right) L \otimes I_{n}\right] x } \\
& =z^{T}\left(U^{T} \otimes I_{n}\right)\left[\sum_{m \in \mathcal{I}} \kappa_{m}\left(\theta_{m}-\Delta_{m}\right) L \otimes I_{n}\right]\left(U \otimes I_{n}\right) z \\
& =\sum_{i=2}^{N} z_{i}^{T}\left[\sum_{m \in \mathcal{I}} \kappa_{m}\left(\theta_{m}-\Delta_{m}\right) \lambda_{i}(L)\right] z_{i} .
\end{aligned}
$$

Similarly

$$
\begin{aligned}
& -c \eta x^{T}\left(L^{2} \otimes I_{n}\right) x \\
& =-c \eta z^{T}\left(U^{T} \otimes I_{n}\right)\left(L^{2} \otimes I_{n}\right)\left(U \otimes I_{n}\right) z \\
& =-c \eta z^{T}\left(U^{T} L^{2} U \otimes I_{n}\right) z \\
& =-c \eta \sum_{i=2}^{N} \lambda_{i}^{2}(L) z_{i}^{T} z_{i} \\
& -D x^{T}\left(L^{2} \otimes I_{n}\right) x=-D \sum_{i=2}^{N} \lambda_{i}^{2}(L) z_{i}^{T} z_{i} .
\end{aligned}
$$

Therefore, substituting (23)-(25) into (22) yields

$$
\begin{aligned}
\mathbb{E} \mathcal{L} V(t)=\mathbb{E} \sum_{i=2}^{N} z_{i}^{T} \lambda_{i}(L) & {\left[\sum_{m \in \mathcal{I}} \kappa_{m}\left(\theta_{m}-\Delta_{m}\right)\right.} \\
& \left.-c \eta \lambda_{i}(L)-D \lambda_{i}(L)\right] z_{i} \leq 0 .
\end{aligned}
$$

According to the inequality in Corollary 3 , the synchronization will be achieved in mean square. This completes the proof.

Furthermore, the following corollary can be obtained.

Corollary 4: Suppose that $g_{m}(x, t),(m \in \mathcal{I})$ is continuous on $(x, t) \in \mathbb{R}^{n} \times \mathbb{R}^{+}$, satisfies Assumptions 1,2 , and 3, graph $\mathcal{G}$ is connected, $\eta_{i}=\eta(i \in \mathcal{V})$ and $c$ is a positive constant, which makes the following inequality hold:

$$
\eta>\frac{\sum_{m \in \mathcal{I}} \kappa_{m}\left(\theta_{m}-\Delta_{m}\right)-D \lambda_{2}(L)}{c \lambda_{2}(L)}
$$

then the network of agent systems (4) under (6) and (8) will be globally synchronized in mean square, and the coupling strengths will converge for any initial data $x_{i}(0) \in \mathbb{R}^{n}, i \in \mathcal{V}$, i.e., $\lim _{t \rightarrow \infty} \epsilon_{i}(t)=\epsilon_{i, \infty}$, where $\epsilon_{i, \infty}(i \in \mathcal{V}) \in \mathbb{R}$ is a constant.

Remark 7: From this corollary, one can obtain the minimum occurrence probability of the controllers in the network. $c$ can be arbitrarily large, and therefore, if the occurrence probability of distributed controllers is nonzero, the synchronization of the network of agent systems (4) can be always achieved in mean square, when $\sum_{m \in \mathcal{I}} \kappa_{m}\left(\theta_{m}-\Delta_{m}\right)-D \lambda_{2}(L)>0$.

If the occurrence probabilities of multiple random controllers are equal in all vertices and only one kind of nonlinearity exists, we have the following results.

Corollary 5: Assume that only one nonlinearity exists in the network of agent systems (4), i.e., $m \in \mathcal{I}=\{1\}$. Suppose that $g_{1}(x, t)$ is continuous on $(x, t) \in \mathbb{R}^{n} \times \mathbb{R}^{+}$, satisfies Assumptions 1, 2, and 3, graph $\mathcal{G}$ is connected, $\eta_{i}=\eta(i \in \mathcal{V})$ and $c$ is a positive constant, which makes the following inequality hold:

$$
\sum_{i=2}^{N}\left[\left(\theta_{1}-\Delta_{1}\right)-c \eta \lambda_{i}(L)-D \lambda_{i}(L)\right]<0
$$

then the network of agent systems (4) under (6) and (8) will be globally synchronized in mean square, and the coupling strengths will converge for any initial data $x_{i}(0) \in \mathbb{R}^{n}, i \in \mathcal{V}$, i.e., $\lim _{t \rightarrow \infty} \epsilon_{i}(t)=\epsilon_{i, \infty}$, where $\epsilon_{i, \infty}(i \in \mathcal{V}) \in \mathbb{R}$ is a constant.

If we utilize the bounds of $\eta_{i},(i \in \mathcal{V})$, the following two corollaries can be obtained by Theorem 1 and Corollary 3 .

Corollary 6: Suppose that $g_{m}(x, t),(m \in \mathcal{I})$ is continuous on $(x, t) \in \mathbb{R}^{n} \times \mathbb{R}^{+}$, satisfies Assumptions 1, 2, and 3, graph $\mathcal{G}$ is connected, and $c$ is a positive constant, which makes the following inequality hold:

$$
\left(\sum_{m \in \mathcal{I}} \kappa_{m} \theta_{m} I_{N}-c \check{\eta} L-D L\right) L \leq 0
$$

then the network of agent systems (4) under (6) and (8) will be globally synchronized in mean square, and the coupling strengths will converge for any initial data $x_{i}(0) \in \mathbb{R}^{n}, i \in \mathcal{V}$, i.e., $\lim _{t \rightarrow \infty} \epsilon_{i}(t)=\epsilon_{i, \infty}$, where $\epsilon_{i, \infty}(i \in \mathcal{V}) \in \mathbb{R}$ is a constant.

Corollary 7: Suppose that $g_{m}(x, t),(m \in \mathcal{I})$ is continuous on $(x, t) \in \mathbb{R}^{n} \times \mathbb{R}^{+}$, satisfies Assumptions 1 , 2, and 3, graph $\mathcal{G}$ is connected, and $c$ is a positive constant, which makes the following inequality hold:

$$
\sum_{i=2}^{N}\left[\sum_{m \in \mathcal{I}} \kappa_{m}\left(\theta_{m}-\Delta_{m}\right)-c \check{\eta} \lambda_{i}(L)-D \lambda_{i}(L)\right]<0
$$

then the network of agent systems (4) under (6) and (8) will be globally synchronized in mean square, and the coupling strengths will converge for any initial data $x_{i}(0) \in \mathbb{R}^{n}$, $i \in \mathcal{V}$, i.e., $\lim _{t \rightarrow \infty} \epsilon_{i}(t)=\epsilon_{i, \infty}$, where $\epsilon_{i, \infty}(i \in \mathcal{V}) \in \mathbb{R}$ is a constant. omitted.

Proof: The proof is similar to Corollary 3 and hence

\section{B. Expectation of Upper Bounds of $\epsilon$ and $\gamma$}

In the following, the expectation of upper bounds of control gain $\epsilon$ and convergence speed $\gamma$ are derived. Here, we further assume that $\check{\alpha}, \check{\eta}$, and $\check{\xi}$ are not equal to 0 . 
Theorem 2: If all assumptions and conditions in Theorem 1 are satisfied, then when $\epsilon_{i}(0)=0,(\forall i \in \mathcal{V})$,

(i) the expectation of the upper bound of $\epsilon$ is

$$
\epsilon \leq \bar{\epsilon}=\mathcal{U}+\sqrt{\mathcal{U}^{2}+\frac{2 p_{0} \hat{\alpha} \hat{\xi}}{\check{\eta} N}}
$$

where $p_{0}=(1 / 4) \sum_{i \in \mathcal{V}} \sum_{j \in \mathcal{N}(i)}\left\|e_{i j}(0)\right\|^{2}, \bar{\alpha}=\hat{\alpha} / \check{\alpha}$, $\bar{\eta}=\hat{\eta} / \check{\eta}, \bar{\xi}=\hat{\xi} / \check{\xi}, \mathcal{U}=c(\bar{\alpha} \bar{\eta} \bar{\xi}-1)+\left(\sum_{m \in \mathcal{I}} \kappa_{m} \theta_{m}-\right.$ $\left.D \lambda_{2}(L) / \lambda_{2}(L) \check{\eta}\right)$. Specifically

$$
\bar{\epsilon} \leq \hat{\epsilon}= \begin{cases}2 \mathcal{U}+\sqrt{\frac{2 p_{0} \hat{\alpha} \hat{\xi}}{\check{\eta} N}}, & \text { if } \mathcal{U} \geq 0 \\ \sqrt{\frac{2 p_{0} \hat{\alpha} \hat{\xi}}{\check{\eta} N}}, & \text { else. }\end{cases}
$$

(ii) Additionally, the expectation of upper bound of $\gamma$ is

$$
\gamma \leq \bar{\gamma}=\frac{N}{(N-1) \lambda_{2}^{2}(L) \check{\alpha} \check{\xi}}\left[\mathcal{U}+\sqrt{\mathcal{U}^{2}+\frac{2 p_{0} \hat{\alpha} \hat{\xi}}{\check{\eta} N}}\right] .
$$

Specifically

$$
\bar{\gamma} \leq \hat{\gamma}= \begin{cases}\frac{N}{(N-1) \lambda_{2}^{2}(L) \check{\alpha} \dot{\xi}}\left[2 \mathcal{U}+\sqrt{\frac{2 p_{0} \hat{\alpha} \hat{\xi}}{\check{\eta} N}}\right], & \text { if } \mathcal{U} \geq 0 \\ \frac{N}{(N-1) \lambda_{2}^{2}(L) \check{\alpha} \dot{\xi}} \sqrt{\frac{2 p_{0} \hat{\alpha} \hat{\xi}}{\check{\eta} N}}, & \text { else. }\end{cases}
$$

Proof: Part I. First, let $v_{i}$ be the eigenvector of $L$ associated with the eigenvalue $\lambda_{i}(L)$ ordered by $0=\lambda_{1}(L) \leq$ $\lambda_{2}(L) \leq \lambda_{3}(L) \leq \ldots \leq \lambda_{N}(L)$. We pick the eigenvectors that correspond to the same eigenvalue with multiplicity such that $v_{1}, \ldots, v_{N}$ compose an orthogonal standard basis of $\mathbb{R}^{N}$. For any $v \in \mathbb{R}^{N}, v$ can be written as $v=\sum_{i \in \mathcal{V}} r_{i} v_{i},(i \in \mathcal{V})$. Thus, one gets $v_{i}^{T} v_{j}=0, \forall i \neq j$

$$
\begin{aligned}
& v^{T}\left\{\left[(c \check{\eta}+D)-\sum_{m \in \mathcal{I}} \frac{\kappa_{m} \theta_{m}}{\lambda_{2}(L)}\right] L^{2}\right. \\
& \left.-\left[(c \check{\eta}+D) L-\sum_{m \in \mathcal{I}} \kappa_{m} \theta_{m} I_{N}\right] L\right\} v \\
& =\sum_{i \in \mathcal{V}} v_{i}^{T} v_{i}\left[\left(c \check{\eta}+D-\sum_{m \in \mathcal{I}} \frac{\kappa_{m} \theta_{m}}{\lambda_{2}(L)}\right) \lambda_{i}^{2}(L)\right. \\
& \left.\quad-(c \check{\eta}+D) \lambda_{i}^{2}(L)+\sum_{m \in \mathcal{I}} \kappa_{m} \theta_{m} \lambda_{i}(L)\right] r_{i}^{2} \\
& +2 \sum_{i \in \mathcal{V}} \sum_{j>i} v_{i}^{T}\left[\left(c \check{\eta}+D-\sum_{m \in \mathcal{I}} \frac{\kappa_{m} \theta_{m}}{\lambda_{2}(L)} L^{2} L^{2}\right.\right. \\
& \left.-\left((c \check{\eta}+D) L-\sum_{m \in \mathcal{I}} \kappa_{m} \theta_{m} I_{N}\right) L\right]
\end{aligned}
$$$$
\times v_{j} r_{i} r_{j}
$$$$
=\sum_{i=2}^{N} \sum_{m \in \mathcal{I}} v_{i}^{T} v_{i}\left[-\frac{1}{\lambda_{2}(L)} \lambda_{i}(L)+1\right] \kappa_{m} \theta_{m} \lambda_{i}(L) r_{i}^{2}
$$$$
\leq 0 \text {. }
$$

By integrating both sides of (8), it yields that

$$
\mathbb{E}\left\{\sum_{i \in \mathcal{V}} \int_{0}^{\infty} \dot{\epsilon}_{i}(t) d t\right\}=\mathbb{E} \sum_{i \in \mathcal{V}} \int_{0}^{\infty} \alpha_{i} \xi_{i}\left[\sum_{j \in \mathcal{N}(i)} e_{i j}\right]^{T}\left[\sum_{j \in \mathcal{N}(i)} e_{i j}\right] d t .
$$

The expectation of $\epsilon$ is obtained from (31)

$$
\mathbb{E}\{\epsilon\}=\mathbb{E}\left\{\frac{1}{N} \int_{0}^{\infty} x^{T}\left(L \mathcal{J} L \otimes I_{n}\right) x d t\right\}
$$

where $\mathcal{J}=\operatorname{diag}\left\{\alpha_{1} \xi_{1}, \ldots, \alpha_{N} \xi_{N}\right\}$. One gets from (32) that

$$
\begin{aligned}
& \mathbb{E}\left\{\frac{\check{\alpha} \check{\xi}}{N} \int_{0}^{\infty} x^{T}\left(L^{2} \otimes I_{n}\right) x d t\right\} \leq \mathbb{E}\{\epsilon\} \\
& \mathbb{E}\{\epsilon\} \leq \mathbb{E}\left\{\frac{\hat{\alpha} \hat{\xi}}{N} \int_{0}^{\infty} x^{T}\left(L^{2} \otimes I_{n}\right) x d t\right\} .
\end{aligned}
$$

From Corollary 6, we get

$$
\mathbb{E} \mathcal{L} V \leq \mathbb{E}\left\{x^{T}(t)\left[\left(\sum_{m \in \mathcal{I}} \kappa_{m} \theta_{m} I_{N}-(c \check{\eta}+D) L\right) L \otimes I_{n}\right] x(t)\right\} .
$$

Thus, combining (17), (30), (32), (34) and (35), one gets

$$
\begin{aligned}
& \mathbb{E}\{\epsilon\}=\mathbb{E}\left\{\frac{1}{N} \int_{0}^{\infty} x^{T}(t)\left(L \mathcal{J} L \otimes I_{n}\right) x(t) d t\right\} \\
& \leq \mathbb{E}\left\{\frac{\hat{\alpha} \hat{\xi} \lambda_{2}(L)}{N\left[(c \check{\eta}+D) \lambda_{2}(L)-\sum_{m \in \mathcal{I}} \kappa_{m} \theta_{m}\right]} \int_{0}^{\infty} x^{T}(t)\right. \\
& \left.\times\left[\left((c \check{\eta}+D) L-\sum_{m \in \mathcal{I}} \kappa_{m} \theta_{m} I_{N}\right) L \otimes I_{n}\right] x(t) d t\right\} \\
& \leq-\mathbb{E}\left\{\frac{\hat{\alpha} \hat{\xi} \lambda_{2}(L)}{N\left[(c \check{\eta}+D) \lambda_{2}(L)-\sum_{m \in \mathcal{I}} \kappa_{m} \theta_{m}\right]}\left[\int_{0}^{\infty} \mathcal{L} V d t\right]\right\} \\
& =\mathbb{E}\left\{\frac{\hat{\alpha} \hat{\xi} \lambda_{2}(L)}{N\left[(c \check{\eta}+D) \lambda_{2}(L)-\sum_{m \in \mathcal{I}} \kappa_{m} \theta_{m}\right]}\left[V_{0}-V_{\infty}\right]\right\} \\
& =\mathbb{E}\left\{\frac{\hat{\alpha} \hat{\xi} \lambda_{2}(L)}{N\left[(c \check{\eta}+D) \lambda_{2}(L)-\sum_{m \in \mathcal{I}} \kappa_{m} \theta_{m}\right]}\right. \\
& \left.\times\left[p_{0}+\sum_{i \in \mathcal{V}} \frac{\eta_{i}}{2 \alpha_{i} \xi_{i}}\left(2 c \epsilon_{i, \infty}-\epsilon_{i, \infty}^{2}\right)\right]\right\} \\
& \leq \mathbb{E}\left\{\frac{\hat{\alpha} \hat{\xi} \lambda_{2}(L)}{N\left[(c \check{\eta}+D) \lambda_{2}(L)-\sum_{m \in \mathcal{I}} \kappa_{m} \theta_{m}\right]}\right. \\
& \left.\times\left[p_{0}+\frac{c N \hat{\eta}}{\check{\alpha} \check{\xi}} \epsilon-\frac{N \check{\eta}}{2 \hat{\alpha} \hat{\xi}} \epsilon^{2}\right]\right\}
\end{aligned}
$$

where $\quad V_{0}=V(0), \quad V_{\infty}=\lim _{t \rightarrow \infty} V(t) \quad$ and $\quad p_{0}=$ $(1 / 4) \sum_{i \in \mathcal{V}} \sum_{j \in \mathcal{N}(i)}\left\|e_{i j}(0)\right\|^{2}$. Denote $\quad \bar{\alpha}=\hat{\alpha} / \check{\alpha}, \bar{\xi}=\hat{\xi} / \check{\xi}$. 
By solving the inequality (36)

$$
\mathbb{E}\{\epsilon\} \leq\{\bar{\epsilon}\}=\mathcal{U}+\sqrt{\mathcal{U}^{2}+\frac{2 p_{0} \hat{\alpha} \hat{\xi}}{\check{\eta} N}}
$$

where

$$
\mathcal{U}=c(\bar{\alpha} \bar{\eta} \bar{\xi}-1)+\frac{\sum_{m \in \mathcal{I}} \kappa_{m} \theta_{m}-D \lambda_{2}(L)}{\lambda_{2}(L) \check{\eta}} .
$$

By using the inequality $\sqrt{a^{2}+b^{2}} \leq a+b$, where $a$ and $b \in$ $\mathbb{R}$ are $a, b \geq 0$

$$
\begin{aligned}
\{\bar{\epsilon}\} & \leq\{\hat{\epsilon}\}=\mathcal{U}+|\mathcal{U}|+\sqrt{\frac{2 p_{0} \hat{\alpha} \hat{\xi}}{\check{\eta} N}} \\
& = \begin{cases}2 \mathcal{U}+\sqrt{\frac{2 p_{0} \hat{\alpha} \hat{\xi}}{\check{\eta} N}}, & \text { if } \mathcal{U} \geq 0 \\
\sqrt{\frac{2 p_{0} \hat{\alpha} \hat{\xi}}{\check{\eta} N}}, & \text { else. }\end{cases}
\end{aligned}
$$

Part II. Next, the expectation of the upper bound of $\gamma$ is given. Let $U=\left(u_{i j}\right)$ with $u_{i j}=-1 / N$ if $i \neq j$ and $u_{i i}=1-1 / N(\forall i \in \mathcal{V})$ and $W=(1 /(N-1)) U^{T} U . \gamma$ can be represented as follows:

$$
\gamma=\int_{0}^{\infty} x^{T}(t)\left(W \otimes I_{n}\right) x(t) d t .
$$

As shown in [48], the following inequality holds:

$$
W \leq \frac{1}{(N-1) \lambda_{2}^{2}(L)} L^{2}
$$

Therefore, we get from (33) and (40) that

$$
\begin{aligned}
\mathbb{E}\{\gamma\} & \leq \mathbb{E} \frac{1}{(N-1) \lambda_{2}^{2}(L)} \int_{0}^{\infty} x^{T}(t)\left(L^{2} \otimes I_{n}\right) x(t) d \\
& \leq \mathbb{E} \frac{N}{(N-1) \lambda_{2}^{2}(L) \check{\alpha} \check{\xi}} \epsilon \\
& \leq \mathbb{E} \frac{N}{(N-1) \lambda_{2}^{2}(L) \check{\alpha} \check{\xi}}\left[\mathcal{U}+\sqrt{\mathcal{U}^{2}+\frac{2 p_{0} \hat{\alpha} \hat{\xi}}{\check{\eta} N}}\right] .
\end{aligned}
$$

Again, by utilizing $\sqrt{a^{2}+b^{2}} \leq a+b$, we have

$$
\{\bar{\gamma}\} \leq \frac{N}{(N-1) \lambda_{2}^{2}(L) \check{\alpha} \check{\xi}}\left[\mathcal{U}+|\mathcal{U}|+\sqrt{\frac{2 p_{0} \hat{\alpha} \hat{\xi}}{\check{\eta} N}}\right] .
$$

Specifically, one has from (42)

$$
\hat{\gamma}= \begin{cases}\frac{N}{(N-1) \lambda_{2}^{2}(L) \check{\alpha} \dot{\xi}}\left[2 \mathcal{U}+\sqrt{\frac{2 p_{0} \hat{\alpha} \hat{\xi}}{\check{\eta} N}}\right], & \text { if } \mathcal{U} \geq 0 \\ \frac{N}{(N-1) \lambda_{2}^{2}(L) \check{\alpha} \dot{\xi}} \sqrt{\frac{2 p_{0} \hat{\alpha} \hat{\xi}}{\check{\eta} N}}, & \text { else. }\end{cases}
$$

This completes the proof.
Remark 8: From Theorem 2, the upper bounds of $\epsilon$ and $\gamma$ depend largely on $\check{\alpha}, \check{\xi}, \check{\eta}, \hat{\alpha}, \hat{\xi}, \kappa_{m}, \theta_{m}$. The nonlinearities and their occurrence probabilities have impacts on the upper bounds of $\epsilon$ and $\gamma$. Note that $c$ can be arbitrarily large which might affect the estimations of $\epsilon$ and $\gamma$. Therefore, in the following, we will develop an algorithm to reduce $c$, and thus the estimations of $\epsilon$ and $\gamma$ will be more exact.

If $\check{\alpha}=\hat{\alpha}=\alpha$ and $\check{\xi}=\hat{\xi}=\xi$, then we have the following theorem.

Theorem 3: If all assumptions and conditions in Theorem 1 are satisfied and $\check{\alpha}=\hat{\alpha}=\alpha, \check{\xi}=\hat{\xi}=\xi$, then when $\epsilon_{i}(0)=$ $0,(\forall i \in \mathcal{V})$,

(i) the expectation of the upper bound of control cost $\epsilon$ is

$$
\epsilon \leq \bar{\epsilon}=\mathcal{X}+\sqrt{\mathcal{X}^{2}+\frac{2 p_{0} \alpha \xi}{\check{\eta} N}}
$$

where $p_{0}=(1 / 4) \sum_{i \in \mathcal{V}} \sum_{j \in \mathcal{N}(i)}\left\|e_{i j}(0)\right\|^{2}$ and

$$
\mathcal{X}=c(\bar{\eta}-1)+\frac{\sum_{m \in \mathcal{I}} \kappa_{m} \theta_{m}-D \lambda_{2}(L)}{\lambda_{2}(L) \check{\eta}} .
$$

Specifically

$$
\bar{\epsilon} \leq \hat{\epsilon}= \begin{cases}2 \mathcal{X}+\sqrt{\frac{2 p_{0} \alpha \xi}{\check{\eta} N}}, & \text { if } \mathcal{X} \geq 0 \\ \sqrt{\frac{2 p_{0} \alpha \xi}{\check{\eta} N}}, & \text { else. }\end{cases}
$$

(ii) Additionally, the expectation upper bound of $\gamma$ is

$$
\gamma \leq \bar{\gamma}=\frac{N}{(N-1) \lambda_{2}^{2}(L)}\left[\mathcal{Y}+\sqrt{\mathcal{Y}^{2}+\frac{2 p_{0} \alpha \xi}{\check{\eta} N}}\right]
$$

where

$$
\mathcal{Y}=\frac{1}{\alpha \xi}\left[c(\bar{\eta}-1)+\frac{\sum_{m \in \mathcal{I}} \kappa_{m} \theta_{m}-D \lambda_{2}(L)}{\lambda_{2}(L) \check{\eta}}\right] .
$$

Specifically

$$
\bar{\gamma} \leq \hat{\gamma}= \begin{cases}\frac{N}{(N-1) \lambda_{2}^{2}(L)}\left[2 \mathcal{Y}+\sqrt{\left.\frac{2 p_{0}}{\alpha \xi \check{\eta} N}\right],}\right. & \text { if } \mathcal{Y} \geq 0 \\ \frac{N}{(N-1) \lambda_{2}^{2}(L)} \sqrt{\frac{2 p_{0}}{\alpha \xi \check{\eta} N}}, & \text { else. }\end{cases}
$$

If $\check{\alpha}=\hat{\alpha}=\alpha, \check{\xi}=\hat{\xi}=\xi$ and $\check{\eta}=\hat{\eta}=\eta$, then we have the following corollary.

Corollary 8: If all assumptions and conditions in Theorem 1 are satisfied and $\check{\alpha}=\hat{\alpha}=\alpha, \check{\xi}=\hat{\xi}=\xi, \check{\eta}=\hat{\eta}=\eta$, then when $\epsilon_{i}(0)=0,(\forall i \in \mathcal{V})$,

(i) the expectation of the upper bound of control cost $\epsilon$ is

$$
\epsilon \leq \bar{\epsilon}=\mathcal{X}+\sqrt{\mathcal{X}^{2}+\frac{2 p_{0} \alpha \xi}{\eta N}}
$$

where $p_{0}=(1 / 4) \sum_{i \in \mathcal{V}} \sum_{j \in \mathcal{N}(i)}\left\|e_{i j}(0)\right\|^{2}$ and

$$
\mathcal{X}=\frac{\sum_{m \in \mathcal{I}} \kappa_{m} \theta_{m}-D \lambda_{2}(L)}{\lambda_{2}(L) \eta} .
$$


Specifically

$$
\bar{\epsilon} \leq \hat{\epsilon}= \begin{cases}2 \mathcal{X}+\sqrt{\frac{2 p_{0} \alpha \xi}{\eta N}}, & \text { if } \mathcal{X} \geq 0 \\ \sqrt{\frac{2 p_{0} \alpha \xi}{\eta N}}, & \text { else. }\end{cases}
$$

(ii) Additionally, the expectation of the upper bound of $\gamma$ is

$$
\gamma \leq \bar{\gamma}=\frac{N}{(N-1) \lambda_{2}^{2}(L)}\left[\mathcal{Y}+\sqrt{\mathcal{Y}^{2}+\frac{2 p_{0} \alpha \xi}{\eta N}}\right]
$$

where

$$
\mathcal{Y}=\frac{\sum_{m \in \mathcal{I}} \kappa_{m} \theta_{m}-D \lambda_{2}(L)}{\lambda_{2}(L) \alpha \xi \eta}
$$

Specifically

$$
\bar{\gamma} \leq \hat{\gamma}= \begin{cases}\frac{N}{(N-1) \lambda_{2}^{2}(L)}\left[2 \mathcal{Y}+\sqrt{\frac{2 p_{0}}{\alpha \xi \eta N}}\right], & \text { if } \mathcal{Y} \geq 0 \\ \frac{N}{(N-1) \lambda_{2}^{2}(L)} \sqrt{\frac{2 p_{0}}{\alpha \xi \eta N}}, & \text { else. }\end{cases}
$$

If $\check{\alpha}=\hat{\alpha}=\alpha, \check{\xi}=\hat{\xi}=\xi, \check{\eta}=\hat{\eta}=\eta$ and there exists only one nonlinearity in (4), then we have the following corollary.

Corollary 9: If all assumptions and conditions in Theorem 1 are satisfied and $\check{\alpha}=\hat{\alpha}=\alpha, \check{\xi}=\hat{\xi}=\xi, \check{\eta}=\hat{\eta}=\eta$, then when $\epsilon_{i}(0)=0,(\forall i \in \mathcal{V})$,

(i) the expectation of the upper bound of control cost $\epsilon$ is

$$
\epsilon \leq \bar{\epsilon}=\mathcal{Z}+\sqrt{\mathcal{Z}^{2}+\frac{2 p_{0} \alpha \xi}{\eta N}}
$$

where $p_{0}=(1 / 4) \sum_{i \in \mathcal{V}} \sum_{j \in \mathcal{N}(i)}\left\|e_{i j}(0)\right\|^{2}$ and

$$
\mathcal{Z}=\frac{\theta_{1}-D \lambda_{2}(L)}{\lambda_{2}(L) \eta}
$$

Specifically

$$
\bar{\epsilon} \leq \hat{\epsilon}= \begin{cases}2 \mathcal{Z}+\sqrt{\frac{2 p_{0} \alpha \xi}{\eta N}}, & \text { if } \mathcal{Z} \geq 0 \\ \sqrt{\frac{2 p_{0} \alpha \xi}{\eta N}}, & \text { else. }\end{cases}
$$

(ii) Additionally, the expectation of the upper bound of $\gamma$ is

$$
\gamma \leq \bar{\gamma}=\frac{N}{(N-1) \lambda_{2}^{2}(L)}\left[\mathcal{K}+\sqrt{\mathcal{K}^{2}+\frac{2 p_{0} \alpha \xi}{\eta N}}\right]
$$

where

$$
\mathcal{K}=\frac{\theta_{1}-D \lambda_{2}(L)}{\lambda_{2}(L) \alpha \xi \eta}
$$

Specifically

$$
\bar{\gamma} \leq \hat{\gamma}= \begin{cases}\frac{N}{(N-1) \lambda_{2}^{2}(L)}\left[2 \mathcal{K}+\sqrt{\frac{2 p_{0}}{\alpha \xi \eta N}}\right], & \text { if } \mathcal{K} \geq 0 \\ \frac{N}{(N-1) \lambda_{2}^{2}(L)} \sqrt{\frac{2 p_{0}}{\alpha \xi \eta N}}, & \text { else. }\end{cases}
$$

Remark 9: From Theorem 3 and Corollaries 8-9, we get that $\eta, \xi, \kappa_{m}, \alpha, D, \lambda_{2}(L)$, and $\theta_{m}$ influence the values of $\epsilon$ and $\gamma$.

\section{NumERICAL EXAMPLES}

In this section, several numerical examples are presented to verify the performance of the proposed MROCs and MROULs for the network of agent systems (4) with MRONs. The effects of $\eta$ and $\xi$ on synchronization are also discussed.

\section{A. Model Description}

A Hopfield neural network is considered on each vertex as an agent [57]

$$
d x(t)=f(x, t) d t=\sum_{m \in \mathcal{I}} \kappa_{m}(t)\left[-C_{m} x+A_{m} h_{m}(x)\right] d t
$$

where $x(t)=\left[x_{1}(t), x_{2}(t), x_{3}(t)\right]^{T}, A_{m}$ and $C_{m}(m \in \mathcal{I})$ are picked as follows:

$$
A_{m}=\left(\begin{array}{ccc}
1.25 & -3.2 & -3.2 \\
-3.2 & 1.1 & -4.4 \\
-3.2 & 4.4 & 1
\end{array}\right), \quad C_{m}=\left(\begin{array}{ccc}
1 & 0 & 0 \\
0 & 1 & 0 \\
0 & 0 & 1
\end{array}\right)
$$

For the sake of simplicity, we consider two cases of nonlinearities, i.e., $q=2$. The activation functions $h_{m}(x)=$ $\left[h_{m}\left(x_{1}\right), h_{m}\left(x_{2}\right), h_{m}\left(x_{3}\right)\right]^{T}$ are chosen as $h_{1}(x)=(|x+1|-$ $|x-1|) / 2$, and $h_{2}(x)=(|x+1|-|x-1|) / 1.8$. The occurrence probabilities $\kappa_{1}$ and $\kappa_{2}$ of nonlinearities are 0.8 and 0.2 , respectively. The simulation time is set as $T=10$. The step size of our algorithm is chosen as 0.001 .

\section{B. Algorithm for Solving the Main Results}

When solving Theorem 1 and for measuring $\epsilon$ and $\gamma$ more exactly, we convert the optimization problem into an SDP problem. Together with Assumption 1 [53], the algorithm is formulated as follows:

$$
\min \sum_{m \in \mathcal{I}} \theta_{m}+c\left\{\begin{array}{c}
\left(\sum_{m \in \mathcal{I}} \kappa_{m} \theta_{m} I_{N}-c L \mathcal{H}-D L\right) L \leq 0 \\
{\left[\begin{array}{cc}
2 \mathcal{B}-\Sigma_{m} * W_{m}^{2}-2 \Delta_{m} & -A_{m} \\
-A_{m}^{T} & \Sigma_{m}
\end{array}\right]<0}
\end{array}\right.
$$

where $\quad \mathcal{B}=\left(C_{m}+\theta_{m} I_{n}\right), \quad m \in \mathcal{I}, \quad \theta_{m}>0, \quad \Sigma_{m}>0$, and $\Delta_{m}>0$.

Remark 10: Although $c$ could be chosen arbitrarily large to ensure the synchronization of the network of agent systems (4), the estimations of the upper bounds of $\epsilon$ and $\gamma$ will be overlarge. Here, the estimations of the upper bounds of $\epsilon$ and $\gamma$ are solved easily by SDP, which is an efficient method to deal with the proposed optimization problem.

\section{Example 1}

First, MROCs (6) and MROULs (8) are used to synchronize the network of agent systems (4) under distributed control. The coupling graph considered here is a scale-free network [58], in which the degree distribution obeys a power law that were observed in many fields. The parameters are set as $\alpha_{i}=\eta_{i}=$ 


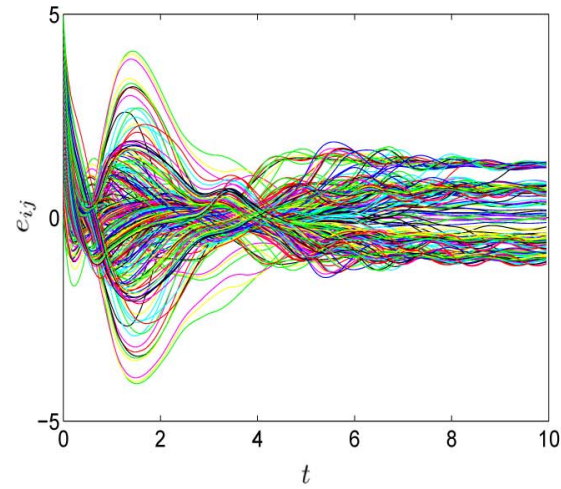

(a)

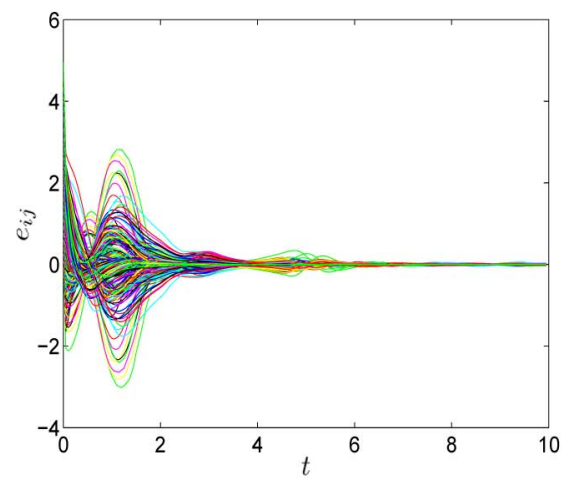

(c)

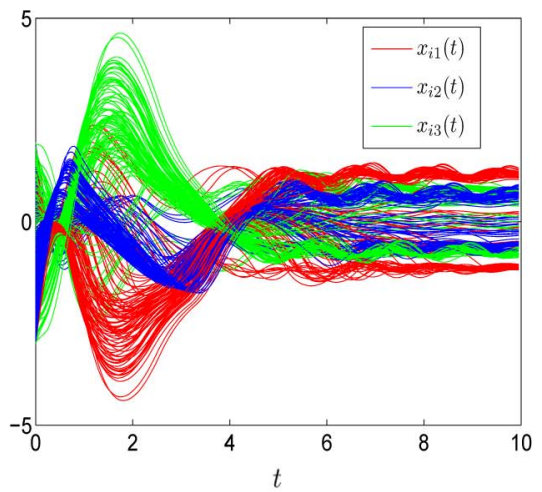

(b)

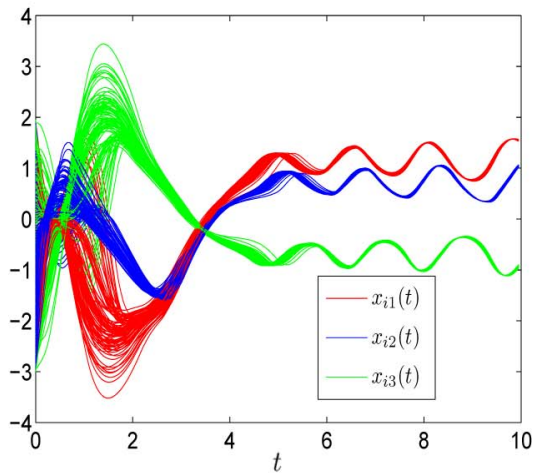

(d)

Fig. 1. Distributed synchronization of the network of agent systems (1) when $N=100, \alpha_{i}=\eta_{i}=\xi_{i}=0.5+0.005 * i, D=0.5$. The figures show the synchronization errors of the network (1) with or without distributed controllers. (a) $x_{1 j}-x_{i j}, i \in \mathcal{V}, j=1, \ldots, n$ without distributed control; (b) $x_{i j}, i \in$ $\mathcal{V}, j=1, \ldots, n$ without distributed control; (c) $x_{1 j}-x_{i j}, i \in \mathcal{V}, j=1, \ldots, n$ under distributed control; (d) $x_{i j}, i \in \mathcal{V}, j=1, \ldots, n$ with distributed control. Fig. 1(a) and (b) shows that synchronization cannot be achieved without distributed control. Fig. 1(c) and (d) illustrates that synchronization can be realized with distributed control.

$\xi_{i}=0.5+0.005 * i, D=0.5, N=100$. It can be checked that $W_{1}=1$ and $W_{2}=10 / 9$.

By using Yalmip [49] to solve (54), the solutions are listed as follows:

$$
\begin{aligned}
c & =11.9947, \Delta_{1}=4.4966 e-004, \theta_{1}=5.5655 \\
\Sigma_{1} & =\operatorname{diag}\{6.6541,7.3625,5.6707\} \\
\Delta_{2} & =8.6447 e-004, \theta_{2}=6.2961 \\
\Sigma_{2} & =\operatorname{diag}\{5.9897,6.6270,5.1049\} .
\end{aligned}
$$

Hence, the distributed synchronization of the network of agent systems (4) with MRONs under MROCs (6) and MROULs (8) is ensured in mean square. When MROCs are not added to the network, the distributed synchronization of the network cannot be achieved, as shown in Fig. 1(a) and (b). The synchronization errors under distributed control are shown in Fig. 1(c) and (d), which shows that synchronization of the network (4) is realized in mean square. Therefore, the criteria developed in this paper are feasible, and the distributed controllers are useful to realize synchronization.

According to Corollary 9, the effects of $\alpha, D, \eta$, and $\xi$ on the control strengths $\epsilon$ and convergence rate $\gamma$ are shown. The network scale here is set as $N=25$. Then, the parameters $\alpha$, $D, \eta, \xi$ are tuned gradually, and their interplays are shown. In Fig. 2, it is shown that the simulation results confirm the bounds of $\epsilon$ and $\gamma$. When $\eta$ increases gradually from 0 to 1 , it is observed that $\epsilon$ and $\gamma$ decrease gradually. However, different from $\eta$, when $\xi$ is adjusted gradually from 0 to 1 , it is found that $\epsilon$ increases and $\gamma$ decreases. The parameter $\alpha$ has the same effect as $\xi$ on $\epsilon$ and $\gamma$. To summarize, the above simulation results show the dependence of $\epsilon$ and $\gamma$ on $\eta$ and $\xi$ well, which is consistent with the corollaries and remarks.

\section{Example 2}

In the second example, we compare the performance of MROCs and the conventional adaptive controller for synchronization of the network of agent systems (4). The traditional adaptive controller is written as follows:

$$
u_{i}(t)=\epsilon_{i}(t)\left(x_{1}(t)-x_{i}(t)\right), \quad i \in \mathcal{V}
$$

and the updating laws are

$$
d \epsilon_{i}(t)=\alpha\left[\left(x_{1}(t)-x_{i}(t)\right)\right]^{T}\left[\left(x_{1}(t)-x_{i}(t)\right)\right] d t .
$$

The parameters are set as follows: $\eta=0.8, \xi=0.8, \alpha=$ $1, D=0.5$. The performance of $\gamma$ when using MROCs (6) is 1.5228, while the performance of $\gamma$ when using the conventional adaptive controller (57) is 2.9841. Although $\epsilon=$ 4.5481 when distributed control is used and $\epsilon=2.8664$ when adaptive control is employed, the distributed control is closer to real-world applications [19]. The results indicate that synchronization of the network of agent systems (4) using MROCs has a faster convergence speed than using the conventional adaptive controller. 


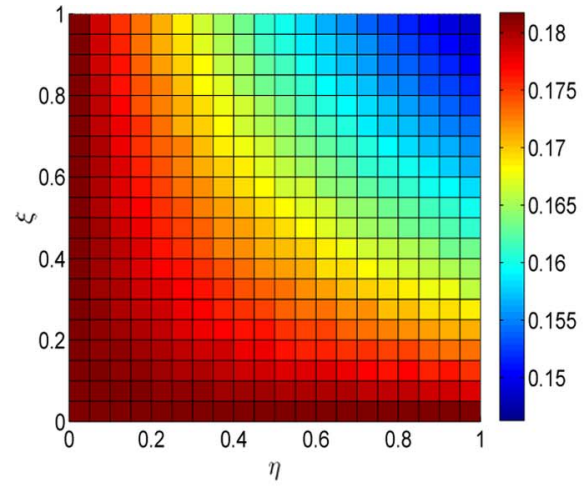

(a)

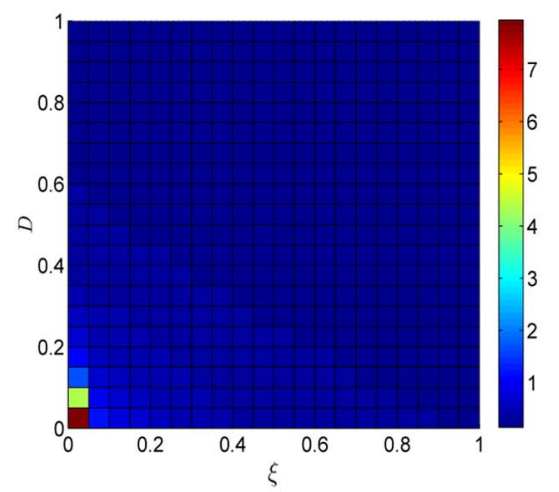

(d)

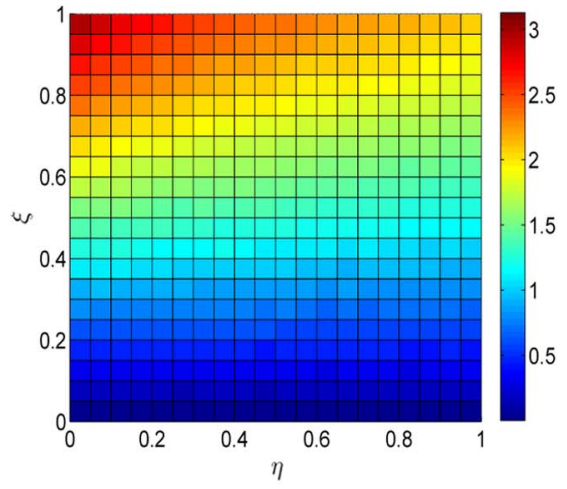

(b)

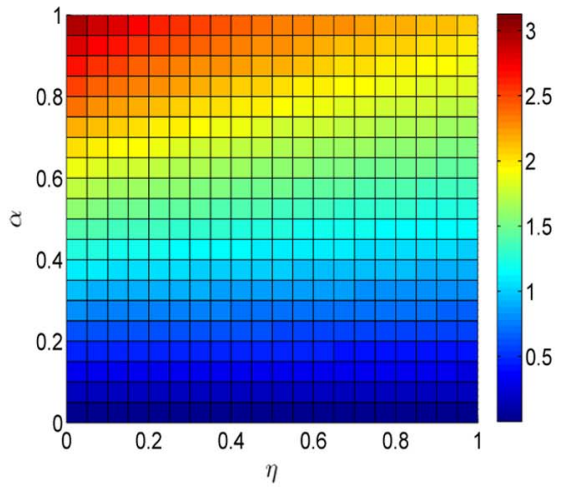

(e)

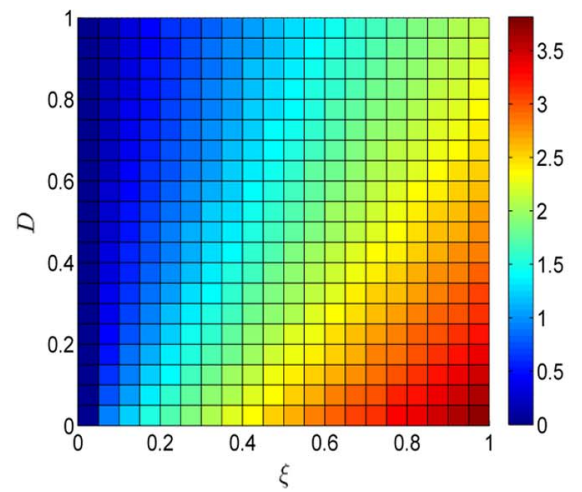

(c)

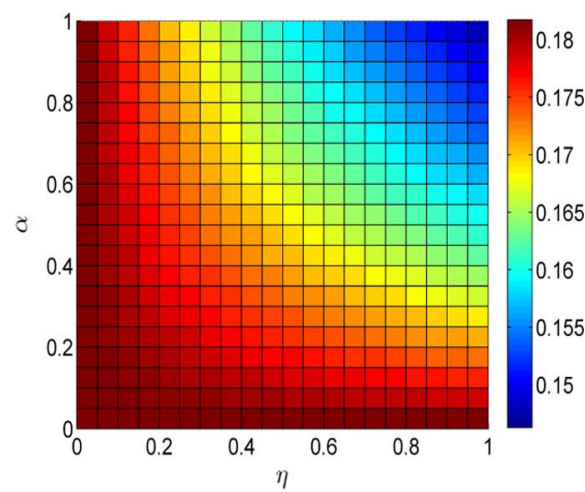

(f)

Fig. 2. Effects of $\alpha, D, \eta$ and $\xi$ on $\epsilon$ and $\gamma$ when $N=25$. (a) Effects of tuning $\eta$ and $\xi$ on $\epsilon$ when $\alpha=1, D=1$; (b) effects of tuning $\eta$ and $\xi$ on $\gamma$ when $\alpha=1, D=1$; (c) effects of tuning $D$ and $\xi$ on $\epsilon$ when $\alpha=1, \eta=1$; (d) effects of tuning $D$ and $\xi$ on $\gamma$ when $\alpha=1, \eta=1$; (e) effects of tuning $\alpha$ and $\eta$ on $\epsilon$ when $\xi=1, D=1$; (f) effects of tuning $\alpha$ and $\eta$ on $\gamma$ when $\xi=1, D=1$. The results verify the theoretical results in Theorems 2 and 3 very well.

\section{CONCLUSION}

We have studied the problem of networks of agent systems via multiple random controllers and nonlinearities. The occurrences of distributed controllers, updating laws of control gain, and nonlinearities are described by three sets of Bernoulli stochastic variables. By utilizing the Lyapunov function method, the distributed synchronization criteria of networks of agent systems under multiple random controllers and probabilistic nonlinearities are presented, which can ensure that synchronization can be achieved in mean square. The presented conditions can be solved by SDP. Furthermore, we show that the overall coupling strength, the probabilities of the Bernoulli stochastic variables, and the form of nonlinearities have significant effects on the convergence speed and the terminal control strength. Specially, the probabilities of occurrence of distributed controllers and updating laws play different roles in terms of convergence speed. The presented synchronization criteria and observed phenomena are confirmed by several numerical simulation examples. In addition, several advantages of distributed adaptive controllers over conventional adaptive controllers are explicitly provided.

In the end, it is valuable to give some further discussions and possible research topics. The first one is to extend our results to synchronization of complex networks or consensus of multi-agent systems with directed and weighted graphs [19], [59]. The second interesting topic is to extend our results to the case of networks with coupling delay [60]-[62]. The third one is to extend our results to two coupled networks [63] or networks of networks [64]. Also, the fourth one is to develop sufficient and necessary conditions under stochastic effects [65] to reduce conservativeness [42], [66]. Finally, we can use some sophisticated intelligent techniques to counteract the effects of nonlinearities [67]-[69].

\section{ACKNOWLEDGMENT}

The authors would like to express their sincere appreciation to the Associate Editor and eight reviewers for their helpful comments.

\section{REFERENCES}

[1] W. Ren and Y. Cao, Distributed Coordination of Multi-Agent Networks, Communications and Control Engineering Series. London, U.K.: Springer-Verlag, 2011.

[2] C. Wu and L. Chua, "Synchronization in an array of linearly coupled dynamical systems," IEEE Trans. Circuits Syst. I, Fundam. Theory Appl., vol. 42, no. 8, pp. 430-447, Aug. 1995.

[3] A. Pikovsky, M. Rosenblum, and J. Kurths, Synchronization: A Universal Concept in Nonlinear Sciences. Cambridge, U.K.: Cambridge Univ. Press, 2001.

[4] A. Arenas, A. Guilera, J. Kurths, Y. Moreno, and C. Zhou, "Synchronization in complex networks," Phys. Rep., vol. 469, no. 3, pp. 93-153, 2008.

[5] H. R. Karimi and P. Maass, "Delay-range-dependent exponential image synchronization of a class of delayed neural networks," Chaos, Solitions Fractals, vol. 41, pp. 1125-1135, 2009.

[6] Y. Tang, Z. D. Wang, W. Wong, J. Kurths, and J. Fang, "Multiobjective synchronization of coupled systems," Chaos, vol. 21, no. 2, p. 025114 , Jun. 2011. 
[7] J. Lu, D. W. C. Ho, J. Cao, and J. Kurths, "Exponential synchronization of linearly coupled neural networks with impulsive disturbances," IEEE Trans. Neural Netw., vol. 22, no. 2, pp. 329-336, Feb. 2011.

[8] H. R. Karimi, "A sliding mode approach to H-infinity synchronization of master-slave time-delay systems with Markovian jumping parameters and nonlinear uncertainties," J. Franklin Inst., vol. 349, no. 4, pp. 1480-1496, May 2012.

[9] J. Liang, Z. Wang, Y. Liu, and X. Liu, "Global synchronization control of general delayed discrete-time networks with stochastic coupling and disturbances," IEEE Trans. Syst., Man, Cybern. B, Cybern., vol. 38, no. 4, pp. 1073-1083, Aug. 2008.

[10] Y. Liu, Z. Wang, J. Liang, and X. Liu, "Synchronization and state estimation for discrete-time complex networks with distributed delays," IEEE Trans. Syst., Man, Cybern. B, Cybern., vol. 38, no. 5, pp. 1314-1325, Oct. 2008.

[11] J. Lu, D. Ho, and Z. Wang, "Pinning stabilization of linearly coupled stochastic neural networks via minimum number of controllers," IEEE Trans. Neural Netw., vol. 20, no. 10, pp. 1617-1629, Oct. 2009.

[12] H. R. Karimi, M. Zapateiro, and N. Luo, "Adaptive synchronization of master-slave systems with mixed neutral and discrete time-delays and nonlinear perturbations," Asian J. Control, vol. 14, no. 1, pp. 251-257, Jan. 2012.

[13] J. Zhou, J. Lu, and J. Lu, "Pinning adaptive synchronization of a general complex dynamical network," Automatica, vol. 44, no. 4, pp. 996-1003, Apr. 2008.

[14] W. Yu, G. Chen, Z. Wang, and W. Yang, "Distributed consensus filtering in sensor networks," IEEE Trans. Syst., Man, Cybern. B, Cybern., vol. 39, no. 6 , pp. $1568-1577$, Dec. 2009.

[15] H. Karimi and H. Gao, "New delay-dependent exponential $h_{\infty}$ synchronization for uncertain neural networks with mixed time delays," IEEE Trans. Syst., Man, Cybern. B, Cybern., vol. 40, no. 1, pp. 173-185, Feb. 2010.

[16] A. Jadbabaie, J. Lin, and A. Morse, "Coordination of groups of mobile autonomous agents using nearest neighbor rules," IEEE Trans. Autom. Control, vol. 48, no. 6, pp. 988-1001, Jun. 2003.

[17] R. Saber and R. Murray, "Consensus problems in networks of agents with switching topology and time-delays," IEEE Trans. Autom. Control, vol. 49, no. 9, pp. 1520-1533, Sep. 2004

[18] W. Ren and R. Beard, "Consensus seeking in multiagent systems under dynamically changing interaction topologies," IEEE Trans. Autom. Control, vol. 50, no. 5, pp. 655-661, May 2005.

[19] R. Saber, J. Fax, and R. Murray, "Consensus and cooperation in networked multi-agent systems," Proc. IEEE, vol. 95, no. 1, pp. 215-233, Jan. 2007.

[20] Z. Li, Z. Duan, and G. Chen, "Consensus of multiagent systems and synchronization of complex networks: A unified viewpoint," IEEE Trans. Circuits Syst. I, Reg. Papers, vol. 57, no. 1, pp. 213-224, Jan. 2010.

[21] Y. Tang, H. Gao, J. Kurths, and J. Fang, "Evolutionary pinning control and its application in UAV coordination," IEEE Trans. Ind. Informat., 2012, to be published.

[22] Y. Tang, Z. D. Wang, and J. Fang, "Pinning control of fractionalorder weighted complex networks," Chaos, vol. 19, no. 1, p. 013112 , Mar. 2009.

[23] J. Zhao, D. J. Hill, and T. Liu, "Stability of dynamical networks with nonidentical nodes: A multiple V-Lyapunov function method," Automatica, vol. 47, no. 12, pp. 2615-2625, Dec. 2011.

[24] W. Zhong, J. D. Stefanovski, G. M. Dimirovski, and J. Zhao, "Decentralized control and synchronization of time-varying complex dynamical network," Kybernetika, vol. 45, no. 1, pp. 151-167, 2009.

[25] L. Wang, Y. Jing, Z. Kong, and G. M. Dimirovski, "Adaptive exponential synchronization of uncertain complex dynamical networks with delay coupling," NeuroQuantology_Int. J. Neurol. Quantum Phys., vol. 6, no. 4, pp. 397-404, 2008.

[26] T. Liu, G. M. Dimirovski, and J. Zhao, "Exponential synchronization of complex delayed dynamical networks with general topology," Phys. A-Statist. Mech. Appl., vol. 387, no. 2/3, pp. 643-652, Jan. 2008.

[27] W. Yu, P. Lellis, G. Chen, M. Bernardo, and J. Kurths, "Distributed adaptive control of synchronization in complex networks," IEEE Trans. Autom. Control, 2012, to be published.

[28] A. Dimakis, S. Kar, J. Moura, M. Rabbat, and A. Scaglione, "Gossip algorithms for distributed signal processing," Proc. IEEE, vol. 98, no. 11, pp. 1847-1864, Nov. 2010 .

[29] W. Yu, G. Chen, M. Cao, and J. Kurths, "Second-order consensus for multi-agent systems with directed topologies and nonlinear dynamics," IEEE Trans. Syst., Man, Cybern. B, Cybern., vol. 40, no. 3, pp. 881-891, Jun. 2010.
[30] P. Lellis, M. Bernardo, and G. Russo, "On QUAD, Lipschitz, and contracting vector fields for consensus and synchronization of networks," IEEE Trans. Circuits Syst. I, Reg. Papers, vol. 58, no. 3, pp. 576-583, Mar. 2011.

[31] J. Hespanha, P. Naghshtabrizi, and Y. Xu, "A survey of recent results in networked control systems," Proc. IEEE, vol. 95, no. 1, pp. 138-162, Jan. 2007.

[32] Z. Wang, Y. Wang, and Y. Liu, "Global synchronization for discrete-time stochastic complex networks with randomly occurred nonlinearities and mixed time-delays," IEEE Trans. Neural Netw., vol. 21, no. 1, pp. 11-25, Jan. 2010.

[33] M. Porfiri and D. Stilwell, "Consensus seeking over random weighted directed graphs," IEEE Trans. Autom. Control, vol. 52, no. 9, pp. 17671773, Sep. 2007.

[34] I. Belykh, V. Belykh, and M. Hasler, "Blinking model and synchronization in small-world networks with a time-varying coupling," Phys. D, vol. 195 , no. 1/2, pp. 188-206, 2004.

[35] B. Shen, Z. Wang, H. Shu, and G. Wei, "H- $\infty$ filtering for uncertain timevarying systems with multiple randomly occurred nonlinearities and successive packet dropouts," Int. J. Robust Nonlin. Control, vol. 21, no. 14, pp. 1693-1709, Sep. 2011

[36] Z. Wang, B. Shen, and X. Liu, "H- $\infty$ filtering with randomly occurring sensor saturations and missing measurements," Automatica, vol. 48, no. 3 , pp. 556-562, Mar. 2012.

[37] X. Sun, G. Liu, D. Rees, and W. Wang, "Stability of systems with controller failure and time-varying delay," IEEE Trans. Autom. Control, vol. 53, no. 10, pp. 2391-2396, Nov. 2008

[38] M. Żochowski, "Intermittent dynamical control," Phys. D, vol. 145 , no. $3 / 4$, pp. $181-190$, Nov. 2000

[39] J. Zhao, D. J. Hill, and T. Liu, "Synchronization of complex dynamical networks with switching topology: A switched system point of view," Automatica, vol. 45, no. 11, pp. 2502-2511, Nov. 2009.

[40] L. Wu, Z. Feng, and W. X. Zheng, "Exponential stability analysis for delayed neural networks with switching parameters: Average dwell time approach," IEEE Trans. Neural Netw., vol. 21, no. 9, pp. 1396-1407, Sep. 2010.

[41] H. Li, B. Chen, Q. Zhou, and W. Qian, "Robust stability for uncertain delayed fuzzy hopfield neural networks with Markovian jumping parameters," IEEE Trans. Syst., Man, Cybern. B, Cybern., vol. 39, no. 1, pp. 94 102, Feb. 2009

[42] M. Huang, S. Dey, G. N. Nair, and J. H. Manton, "Stochastic consensus over noisy networks with Markovian and arbitrary switches," Automatica, vol. 46, no. 10, pp. 1571-1583, Oct. 2010.

[43] Z. Wang, F. Yang, D. Ho, and X. Liu, "Robust $\mathrm{H}-\infty$ control for networked systems with random packet losses," IEEE Trans. Syst., Man, Cybern. B, Cybern., vol. 37, no. 4, pp. 916-924, Aug. 2007.

[44] L. Wu, X. Yao, and W. X. Zheng, "Generalized $\mathrm{H}_{2}$ fault detection for Markovian jumping two-dimensional systems," Automatica, 2012, to be published.

[45] H. Gao, X. Meng, and T. Chen, "Stabilization of networked control systems with a new delay characterization," IEEE Trans. Autom. Control, vol. 53, no. 9, pp. 2142-2148, Oct. 2008.

[46] H. Gao, Y. Zhao, and T. Chen, "H- $\infty$ fuzzy control of nonlinear systems under unreliable communication links," IEEE Trans. Fuzzy Syst., vol. 17, no. 2, pp. 265-278, Apr. 2009.

[47] L. Xiao, S. Boyd, and S. Kim, "Distributed average consensus with least-mean-square deviation," J. Parallel Distrib. Comput., vol. 67, no. 1, pp. 33-46, 2007.

[48] W. Lu, "Adaptive dynamical networks via neighborhood information: Synchronization and pinning control," Chaos, vol. 17, no. 2, p. 023 122, Jun. 2007.

[49] J. Lofberg, "YALMIP: A toolbox for modeling and optimization in MATLAB," in Proc. CACSD Conf., Taipei, Taiwan, 2004, pp. 284-289.

[50] W. Yu, G. Chen, and M. Cao, "Consensus in directed networks of agents with nonlinear dynamics," IEEE Trans. Autom. Control, vol. 56, no. 6 , pp. 1436-1441, Jun. 2011.

[51] D. Yue, E. Tian, Z. Wang, and J. Lam, "Stabilization of systems with probabilistic interval input delays and its applications to networked control systems," IEEE Trans. Syst., Man, Cybern., A, Syst. Humans, vol. 39, no. 4, pp. 939-945, Jul. 2009.

[52] G. Wei, Z. Wang, and H. Shu, "Robust filtering with stochastic nonlinearities and multiple missing measurements," Automatica, vol. 45, no. 3 , pp. 836-841, Mar. 2009.

[53] W. Lu and T. Chen, "New approach to synchronization analysis of linearly coupled ordinary differential systems," Phys. D, vol. 213, no. 2, pp. 214230, Jan. 2006 
[54] X. Mao and C. Yuan, Stochastic Differential Equations With Markovian Switching. London, U.K.: Imperial College Press, 2008.

[55] X. Mao, "Exponential stability of stochastic delay interval systems with Markovian switching," IEEE Trans. Autom. Control, vol. 47, no. 10, pp. 1604-1612, Oct. 2002.

[56] R. Horn and C. Johnson, Matrix Analysis. Cambridge, U.K.: Cambridge Univ. Press, 1990.

[57] F. Zou and J. Nossek, "Bifurcation and chaos in cellular neural networks," IEEE Trans. Circuits Syst. I, Fundam. Theory Appl., vol. 40, no. 3, pp. 166-173, Mar. 1993.

[58] A. Barabási and R. Albert, "Emergence of scaling in random networks," Science, vol. 286, no. 5439, pp. 509-512, Oct. 1999.

[59] J. Qin, W. Zheng, and H. Gao, "Consensus of multiple second-order vehicles with a time-varying reference signal under directed topology," Automatica, vol. 47, no. 9, pp. 1983-1991, Sep. 2011.

[60] W. Zou, J. Lu, Y. Tang, C. Zhang, and J. Kurths, "Control of delay-induced oscillation death by coupling phase in coupled oscillators," Phys. Rev. E, vol. 84, no. 6, p. 066 208, Dec. 2011.

[61] H. R. Karimi, "Robust delay-dependent $h_{\infty}$ control of uncertain Markovian jump systems with mixed neutral, discrete and distributed time-delays," IEEE Trans. Circuits Syst. I, Reg. Papers, vol. 58, no. 8, pp. 1910-1923, Aug. 2011.

[62] H. Li, H. Gao, and P. Shi, "New passivity analysis for neural networks with discrete and distributed delays," IEEE Trans. Neural Netw., vol. 21, no. 11, pp. 1842-1847, Nov. 2010.

[63] C. Li, W. Sun, and J. Kurths, "Synchronization between two coupled complex networks," Phys. Rev. E, vol. 76, no. 4, p. 046 204, Oct. 2007.

[64] J. Donges, H. Schultz, N. Marwan, Y. Zou, and J. Kurths, "Investigating the topology of interacting networks: Theory application to coupled climate subnetworks," Eur. Phys. J. B, vol. 84, no. 4, pp. 635-651, 2011.

[65] Y. Tang, W. Zou, J. Lu, and J. Kurths, "Stochastic resonance in an ensemble of bistable systems under stable distribution noises and heterogeneous coupling," Phys. Rev. E, vol. 85, no. 4, p. 046207 , Apr. 2012.

[66] L. Cheng, Z. Hou, M. Tan, and X. Wang, "Necessary and sufficient conditions for consensus of double-integrator multi-agent systems with measurement noises," IEEE Trans. Autom. Control, vol. 56, no. 8, pp. 1958-1963, Aug. 2011.

[67] Z. Hou, L. Cheng, and M. Tan, "Decentralized robust adaptive control for the multiagent system consensus problem using neural networks," IEEE Trans. Syst., Man, Cybern. B, Cybern., vol. 39, no. 3, pp. 636-647, Jun. 2009.

[68] L. Cheng, Z. Hou, M. Tan, Y. Lin, and W. Zhang, "Neural-network-based adaptive leader-following control for multi-agent systems with uncertainties," IEEE Trans. Neural Netw., vol. 21, no. 8, pp. 1351-1358, Aug. 2010.

[69] G. Chen and F. L. Lewis, "Distributed adaptive tracking control for synchronization of unknown networked Lagrangian systems," IEEE Trans. Syst., Man, Cybern. B, Cybern., vol. 41, no. 3, pp. 805-816, Jun. 2011.

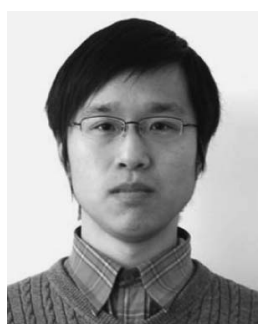

Yang Tang (M'11) received the B.S. and Ph.D. degrees in electrical engineering from Donghua University, Shanghai, China, in 2006 and 2011, respectively.

From December 2008 to December 2010, he was a Research Associate in The Hong Kong Polytechnic University, Hung Hom Kowloon, Hong Kong, China. Now, he is an Alexander von Humboldt Research Fellow at Humboldt University, Berlin, Germany, since 2011. He held Visiting Fellow position at Brunel University in the U.K. from May 2012 to June 2012. He has published more than 20 refereed international journals. His main research interests are synchronization/consensus, networked control system, evolutionary computation, bioinformatics and their applications. He is a very active reviewer for many international journals.

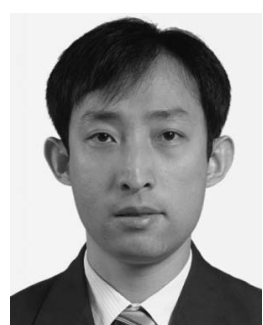

Huijun Gao (SM'09) received the Ph.D. degree in control science and engineering from Harbin Institute of Technology, Harbin, China, in 2005.

He was a Research Associate with the Department of Mechanical Engineering, The University of Hong Kong, from November 2003 to August 2004. From October 2005 to October 2007, he carried out his Postdoctoral Research with the Department of Electrical and Computer Engineering, University of Alberta, Canada. Since November 2004, he has been with Harbin Institute of Technology, where he is currently a Professor and Director of the Research Institute of Intelligent Control and Systems. He research interests include network-based control, robust control/filter theory, time-delay systems, and their engineering applications. He is an Associate Editor for Automatica, IEEE TRANSACTIONS ON Industrial Electronics, IEEE Transactions on Systems, Man, AND CYBERnETICS PART B: CyBERNETICS, IEEE TRANSACTIONS ON FUZZY SySTEMS, IEEE TRANSACTIONS ON CIRCUITS AND SYSTEMS-I, IEEE TRANSACTIONS ON CONTROL SySTEMS TEChNOLOGY, etc.

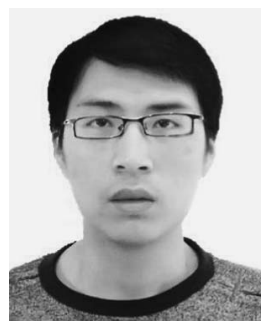

Wei Zou received the B.S. degree from Wuhan University of Science and Technology, Wuhan, China, in 2005, and the Ph.D. degree from Wuhan Institute of Physics and Mathematics, Chinese Academy of Sciences, Wuhan, China, in 2010, respectively.

Currently, he is a Lecturer with the School of Mathematics and Statistics, Huazhong University of Science and Technology, Wuhan, China. He is the author/coauthor of more than 15 international journals. He is the Recipient of Excellent Doctoral Dissertation of Chinese Academy of Science, in 2011. $\mathrm{He}$ is an Alexander von Humboldt Research Fellow at Humboldt University, Berlin, Germany, since 2011. His current research interests include collective behavior of complex networks, dynamics of delay-coupled nonlinear oscillators, and controlling spatiotemporal chaos.

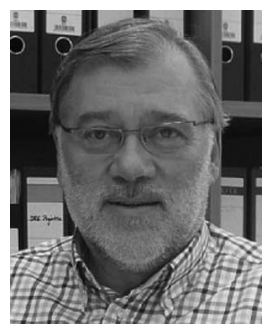

Jürgen Kurths studied mathematics from the University of Rostock, Rostock, Germany, and received the Ph.D. degree from the GDR Academy of Sciences, in 1983.

$\mathrm{He}$ was full Professor from the University of Potsdam, Potsdam, Germany, from 1994 to 2008, has been a Professor of Nonlinear Dynamics at the Humboldt University, Berlin, Germany, the Chair of the Research Domain Transdisciplinary Concepts of the Potsdam Institute for Climate Impact Research since 2008, and a 6th-century Chair of Aberdeen University, Aberdeen, U.K., since 2009. He is a Fellow of the American Physical Society. He has become a member of the Academia Europaea, in 2010, and of the Macedonian Academy of Sciences and Arts, in 2012. His main research interests are synchronization, complex networks, time series analysis, and their applications. He has published more than 480 papers which are cited more than 18000 times (H-factor: 56). He is an Editor for PLoS ONE, Philosophical Transaction of The Royal Society A, CHAOS etc.

Prof. Kurths got an Alexander von Humboldt research award from CSIR (India), in 2005, a Honorary Doctorate, in 2008, from the Lobachevsky University Nizhny Novgorod and one, in 2012, from the State University Saratov. 